\title{
Emerging Science and Research Opportunities for Metals and Metallic Nanostructures
}

\author{
CAROL A. HANDWERKER ${ }^{1,3}$ and TRESA M. POLLOCK ${ }^{2}$ \\ 1.-School of Materials Engineering, Purdue University, West Lafayette, IN 47907, USA \\ 2.-Materials Department, University of California Santa Barbara, Santa Barbara, CA 93106, \\ USA. 3.—e-mail: handwerker@purdue.edu
}

During the next decade, fundamental research on metals and metallic nanostructures (MMNs) has the potential to continue transforming metals science into innovative materials, devices, and systems. A workshop to identify emerging and potentially transformative research areas in MMNs was held June 13 and 14, 2012, at the University of California Santa Barbara. There were 47 attendees at the workshop (listed in the Acknowledgements section), representing a broad range of academic institutions, industry, and government laboratories. The metals and metallic nanostructures (MMNs) workshop aimed to identify significant research trends, scientific fundamentals, and recent breakthroughs that can enable new or enhanced MMN performance, either alone or in a more complex materials system, for a wide range of applications. Additionally, the role that MMN research can play in high-priority research and development (R\&D) areas such as the U.S. Materials Genome Initiative, the National Nanotechnology Initiative, the Advanced Manufacturing Initiative, and other similar initiatives that exist internationally was assessed. The workshop also addressed critical issues related to materials research instrumentation and the cyberinfrastructure for materials science research and education, as well as science, technology, engineering, and mathematics (STEM) workforce development, with emphasis on the United States but with an appreciation that similar challenges and opportunities for the materials community exist internationally. A central theme of the workshop was that research in MMNs has provided and will continue to provide societal benefits through the integration of experiment, theory, and simulation to link atomistic, nanoscale, microscale, and mesoscale phenomena across time scales for an ever-widening range of applications. Within this overarching theme, the workshop participants identified emerging research opportunities that are categorized and described in more detail in the following sections in terms of the following: three-dimensional (3-D) and fourdimensional (4-D) materials science. Structure evolution and the challenge of heterogeneous and multicomponent systems. The science base for property prediction across the length scales. Nanoscale phenomena at surfaces-experiment, theory, and simulation. Prediction and control of the morphology, microstructure, and properties of "bulk" nanostructured metals. Functionality and control of materials far from equilibrium. Hybrid and multifunctional materials assemblies. Materials discovery and design: enhancing the theory-simulation-experiment loop. Following an introduction, these emerging research opportunities are discussed in detail, along with challenges and opportunities for the materials community in the areas of instrumentation, cyberinfrastructure, education, and workforce development. 


\section{INTRODUCTION: GRAND CHALLENGES FOR METALS AND METALLIC NANOSTRUCTURES}

Fundamental research in MMNs has led to some of the most profound science underlying the structure, processing and properties of materials. Linking atomic-level interactions to interface motion, defect formation, and phase transformations has allowed us to transform metals and metal alloys into nanostructures, microstructures, and mesostructures, with exceptional control, performance, and stability. This research has transformed more than just the science and technologies directly associated with MMN. It has provided the basis for understanding structure-dependent properties and processing of ceramics, semiconductors, composites, and complex heterogeneous material structures across broadening length and time scales. The National Science Foundation (NSF) has played a major role in creating this knowledge and educating the field's leaders by "supporting fundamental research on the relationship between the structure and properties of metals and alloys"..."encompassing length scales from the atomic to the macroscopic, including the nano, micro and mesoscales."

During the next decade, fundamental research in MMNs has the potential to continue to produce new science and transform it into innovative materials, devices, and systems. Metals are everywhere, from steel, aluminum, and titanium for transportation and infrastructure to shape-memory alloys for biomedical implants and metal nanoparticles as carriers for cancer detection and treatment. Although it is common to think of these only at the macroscale in terms of their applications, all of these require control at the nanoscale to create and improve the properties for which they are known. MMN research reveals the physical and chemical laws governing phase transformations, the structures they create, and their resulting properties, and it integrates this knowledge into theory, computation and simulation, and materials design. While each application targets specific properties and performance, whether functional, structural, or biological, fundamental metals science creates a path for their design, manufacture, and use.

The societal and economic impacts of MMN research will continue to be significant. MMNs underlie systems and technologies critical to solving national and global grand challenges. Innovations in metals, metal alloys, and metallic nanostructures are needed to advance systems in the following areas:

- Energy generation, harvesting, and storagethermoelectrics, batteries, fuel cells, advanced turbines, magnetic induction, motors, hydrogen production and storage, nuclear reactor materials, and separation membranes
- Propulsion and transportation-high-strength, high-performance, lightweight structures; materials designed for improved creep, impact, fatigue, or corrosion resistance; high-temperature metals, alloys, and coatings for extreme environments; nondestructive evaluation

- Electronics, telecommunication, and information technology—electronic packaging, process modeling, device nanostructures to overcome the end to Moore's law, thin film and nanostructured electrical interconnects, magnetic devices and motors, and sensors

- Sustainability-nanostructured metals for catalytic convertors, polymer catalysis, hydrogen and ammonia production, and low-pollution smelting and refining processes

- Health care-imaging systems; integrated in vivo sensors, electronics, and communication systems for health monitoring and drug delivery; prosthetics and implant materials and systems; and cancer treatment technologies

- Manufacturing-additive and nanomanufacturing technologies, nonequilibrium processing approaches, recycling and reuse, and rapid deployment of alternatives to "critical" materials

Fundamental and applied research in MMNs is continuing to play a leading role in creating the informatics and simulation-based materials-innovation infrastructure embodied in the U.S. Federal R\&D Materials Genome Initiative (MGI) and the National Nanotechnology Initiative (NNI). The MGI was established in 2011 to accelerate the pace of materials development and leverage synergies between experimental and computational materials science and engineering research across multiple federal agencies. The central premise of MGI is that the period between the discovery of materials and their integration into new technologies and applications will be shortened by creating greater synergy between experimental and computational research and developing the infrastructure to support this translation. Likewise, the NNI is developing the scientific knowledge base for nanotechnology across materials systems, applications, and STEM disciplines, and it is translating this research into nanoenabled products, a scientific workforce trained in nanotechnology, and societal benefits as diverse as improved health care and national security.

Sponsored by the National Science Foundation, the Workshop was held in June 2012 to identify significant research trends, fundamental science directions, and recent breakthroughs in MMNs that will strengthen the ability to respond to these scientific challenges and national priorities. A central theme of the Workshop was that research in MMNs has provided and will continue to provide broad societal benefits through the combination of experiment, theory, and simulation to link atomistic, nanoscale, microscale, and mesoscale phenomena at various time scales for an everwidening range of applications. The design of new 
metal systems and metallic nanostructures requires an understanding of the processes used to create them, their evolution over time, and their complex/realistic geometries in three dimensions. As identified in the Workshop, emerging theory, simulation, and experimental research approaches in MMNs rely on advanced synthesis techniques, computational and characterization tools with unparalleled spatial and temporal resolution, and their integration for bridging length and time scales. Effective education remains necessary for effective research and its promulgation into materials and systems. In addition to covering the full spectrum of materials education, undergraduate and graduate curricula must continue to evolve and integrate materials science with design of engineering systems to better prepare the workforce in supporting, advancing, and applying MMN research approaches to ever-broadening applications. In developing this future workforce, it will also be critical to create a community that nurtures early career scientists, particularly academics.

In the following sections, we outline specific scientific opportunities enabled by emerging research in MMNs. These emerging opportunities are not meant to be all inclusive but to illustrate the intellectual promise of MMN research, as well as its widening impact. Additionally, critical issues limiting the materials research infrastructure, education, and workforce development are addressed.

\section{EMERGING SCIENCE AND NATIONAL PRIORITIES IN METALS AND METALLIC NANOSTRUCTURES}

\section{Three-Dimensional and 4-D Materials Science}

Control of structure across all length scales and over time is at the core of the design and discovery process for new metallic systems with unique functionality. In the context of materials science, the term "structure" at its most basic level encompasses not only the atomistic details of single-phase materials but also multiple phases, with their compositions, crystal structures, morphologies, crystallographic orientations, states of stress, and defects. The next level of "structure" encompasses the spatial relationship of these multiple phases and their interfaces, and their evolution in time and space to create real materials with properties dependent on a wide range of material length scales. The embodiment of structure and structure evolution into models of material function and properties is an urgent need for meeting current research priorities, such as MGI and NNI. Predicting and characterizing structure in three dimensions from the nanoscale to the mesoscale and its evolution over timescales ranging from picoseconds to multiple years (known as 4-D materials science) will require major scientific advances along all fronts, from new modeling and experimental approaches to new instrumentation with the requisite spatial and temporal resolution. Because modeling and measurement of structure and structure evolution require multidimensional representation, usually across multiple researchers, these advances must be accompanied by an increasing ability to effectively and efficiently collect, integrate, analyze, and share terabyte-scale data sets to capture materials structure in three and four dimensions. Through this approach, the combination of theory, experiments, and modeling should be strengthened and the feedback loop shortened. Innovative modes for collaboration and use of unique (and usually expensive) instrumentation (with their complex data sets) will also need to be created and tested, and the education process for materials students to learn to use these approaches and tools will need to be expanded and enhanced.

Newly emerging tomography techniques offer unprecedented levels of resolution and insight into material structure across the length scales. ${ }^{1}$ At the high-resolution end of the structural spectrum, there have been remarkable developments in instrumentation for 3-D and 4-D materials science, including local electrode atom probe tomography (LEAP) atom probe tomography and transmission electron microscopy (TEM)-based electron tomography (including scanning transmission electron microscopy (STEM) with angular dark-field imaging and vector field electron tomography, as well as with conventional bright-field and dark- field imaging modes), hybrid environmental scanning electron microscopy (ESEM)-STEM tomography, and dynamic transmission electron microscopy (DTEM). ${ }^{2-6}$ At higher length scales, synchrotron and laboratory-scale $\mathrm{x}$-ray tomography using $\mathrm{x}$-ray absorption, diffraction, and phase contrast mechanisms now permit the acquisition of structural, crystallographic, and stress state information. ${ }^{7-9}$ For structural, chemical, and crystallographic information over larger material volumes, new capabilities in the areas of focused-ion beam (FIB) tomography, automated mechanical serial sectioning, and femtosecond laser-assisted tomography have also evolved dramatically. ${ }^{10-16}$ These techniques, taken individually and in combination, are transforming the scientific linkages among processing, structure, and properties, permitting radical improvements in our ability to explain the processing and structural origins of a wide range of material properties. Examples of selected 3-D data sets shown at the workshop are given in Fig. $1 .{ }^{17-20}$ These data sets illustrate the rich diversity of scientific challenges for metals structure research: void formation during processing of metal matrix composites, composition and phase distributions in zirconium alloys for nuclear fuel rod cladding, the character of grain boundary networks that result in crack initiation, and dislocation distributions in a Mo nanopillar.

Emerging 3-D and 4-D characterization techniques also bring a range of challenges with regard to the large size of the resulting data sets, the fundamental issues regarding image reconstruction, and the ability to integrate data from multiple instrument sources into a complex representation of structure evolution. ${ }^{21}$ Synchrotron experiments can easily generate terabyte-size data sets and, other than high-capacity magnetic recording devices, there is no straightfor- 
Atom Probe Tomography of Oxides on $\mathrm{Zr}$

(a)

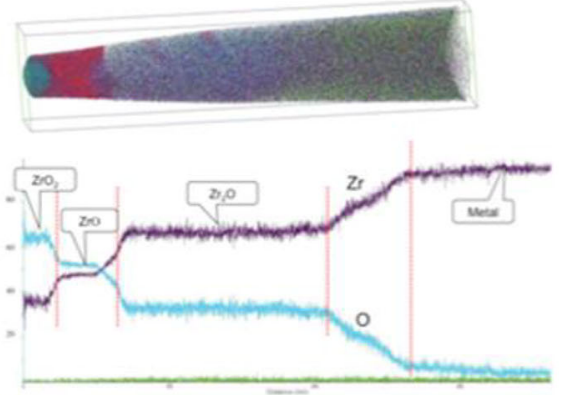

FIB Tomography of Grain Boundary Networks

(c)
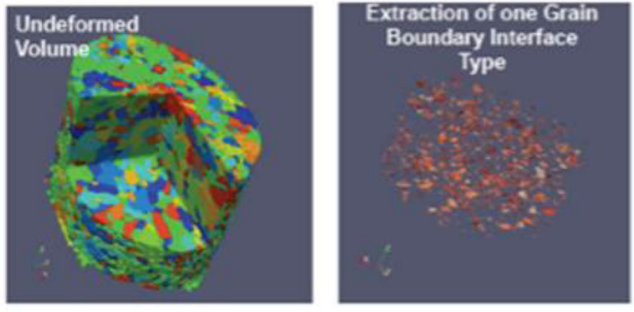

(d)

SiC Particles and Voids at Fracture

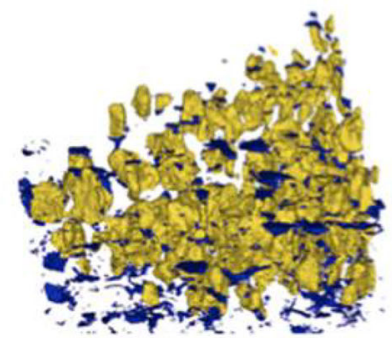

(b)

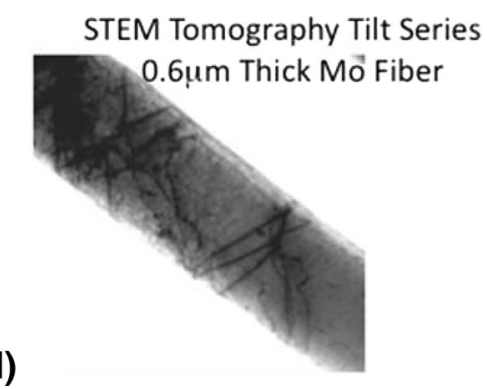

Fig. 1. Examples of 3-D materials information: (a) composition and phases in zirconium alloys for nuclear fuel rod cladding, ${ }^{18}$ (b) void formation during processing of metal matrix composites, ${ }^{17}$ (c) the character of grain boundary networks that result in crack initiation, ${ }^{19}$ and (d) dislocation distributions in a Mo nanopillar. ${ }^{20}$

ward manner to transfer or share this large volume of raw data. Although it is often recognized that sample fabrication and data acquisition require substantial effort, the segmentation and reconstruction processes often require even more. Large data sets in x-ray, electron, or optical approaches are collected with instrument operating parameters that are often selected without consideration of the challenges of segmentation and reconstruction. For any instrument, the images gathered are a convolution of the true image, noise in the instrumentation system, the signal measured by the detector, and the algorithms used to extract the data from the signal. Reconstruction approaches that explicitly consider the physics-based instrument point spread functions simultaneously with data collection could radically improve the speed of reconstruction and the quality of information gathered. Reconstruction approaches are also needed for integrating disparate data sets to recreate a microstructure. This could be done, for example, by fusion of volumetric strain and crystallographic information from x-ray diffraction measurements with twodimensional (2-D) multislice information on crystallographic orientation, microstructure, and composition data sets obtained from scanning electron microscopy (SEM)-based electron back scattered diffraction (EBSD) and energy dispersive x-ray spectroscopy (EDS) analyses. Fundamental challenges still exist on the statistics of structure and the determination of the physical size of the representative volume element (RVE) that must be probed for the prediction of a given property. Whereas the RVE is typically considered as the smallest volume element of a material that can reproduce a macroscopic constitutive response, for properties that derive from a highly local response within a materials structure, the RVE is not yet well defined. For example, it may be possible to define an RVE for the assessment of the elastic properties of a heterogeneous material but not the fatigue strength or fracture toughness of the same material. Furthermore, mathematically rigorous, automated algorithms and workflows for meshing and analyzing complex microstructural and nanoscale features are needed to make the linkage to structure and evolving properties.

The volume of data that will be acquired by emerging, and as yet to be developed 3-D and 4-D techniques, is potentially enormous. All the relevant data for any materials system will be heterogeneous and, in many cases, distributed internationally across a range of institutions with specialized instrumentation. In this sense, "big data" challenges exist on all fronts, including the capture, representation, analysis, and storage of the $3-\mathrm{D}$ and 4-D data. Resolving these challenges will require the combined expertise, collaboration, and support of multidisciplinary teams. The hub model for collaborating and sharing of information, notably embodied by the NSF-supported nanoHub ${ }^{22}$ and MatForge (www.matforge.org), has already demonstrated how some of the challenges in sharing simulation software, data, methods, and educational tools can be addressed to support the research community. There are also important efforts to develop community-supported software tools for 3-D microstructure science such as, Dream.3D (http:// dream3d.bluequartz.net). 


\section{Structure Evolution and the Challenge of Heterogeneous and Multicomponent Systems}

The fundamental tenet that properties are determined by composition and structure demands that their evolution be predictable from materials design through the earliest stages of material processing and manufacturing and into service environments. The vastly varying timescales of structural evolution processes pose major challenges for full experimental characterization. These evolution processes include nucleation from the solid, liquid, vapor, and plasma states; growth of distinct domains, phases (stable and metastable), and grains; and longer-timescale processes, such as coarsening and evolution of structure due to externally imposed mechanical, chemical, or electrical fields. Models for structure formation and evolution are thus essential but are strongly challenged by the multicomponent character of the majority of metallic systems in use.

At the core of the structural evolution problem is the ability to predict behavior at the atomistic scale and make connections to the continuum-scale descriptions. This requires fundamental knowledge of the thermodynamics and kinetics of transformations, which in turn demands accurate thermodynamic models of multicomponent systems that are captured in validated databases. This has long been recognized by the CALPHAD community, ${ }^{22-25}$ although thermodynamic databases, while now commercially available, are incomplete. In part, this stems from the slow process of generating and assessing experimental data and developing and validating models for the Gibbs free energy of multicomponent compounds that often depart from stoichiometry and contain defects. The process of experimentally mapping out these databases can itself lead to new materials discoveries. For example, a rigorous thermodynamic evaluation of the $\mathrm{Co}-\mathrm{Al}-$ $\mathrm{W}$ ternary diagram in $2006^{26}$ resulted in the discovery of a new ternary $\mathrm{L}_{2}$ phase, $\mathrm{Co}_{3}(\mathrm{Al}, \mathrm{W})$, which shows promise as a major constituent of metallic single crystals for high-temperature applications. ${ }^{27}$ Other multicomponent metallic systems suffer from incomplete knowledge of ternary and quaternary phase equilibria and of the many intermetallic compounds that could provide pathways for improving the functionality and structural performance of metal systems. Of particular note in this regard are alloys based on magnesium, which is the structural metal with not only the lowest density but also excellent biocompatibility and resorbability for biomedical implants. ${ }^{28}$

Beyond the need for accurately modeling the thermodynamics of multicomponent systems is a demand for models and data of atomic and interface/ grain boundary mobility and transformation kinetics to predict diffusion and structure evolution. ${ }^{24}$ First-principles analyses combined with experimental information is likely the most efficient path forward for thermodynamic and kinetic analyses of bulk materials. ${ }^{29,30}$ The materials dominated by the presence of high densities of interfaces, discussed in more detail later, present even greater challenges.

Ultimately, the challenge, which is summarized in Fig. $2^{33}$ is to develop a computational framework that integrates atomistics, thermodynamics (including thermodynamic quantities beyond composition), and kinetics for bulk materials as well as their interfaces. Such a framework must capture not only the features that determine important average properties but also those at the tails of the structural and compositional distributions that determine performance. Therefore, many aspects of structure evolution must also be addressed at the mesoscale. This includes, for example, the size, shape, spatial and orientation distributions of precipitates, size and morphology of grains, or size, shape, and spatial distribution of pores in porous materials. These structures are exquisitely dependent on the processing paths used to produce them, and these paths frequently involve time scales far in excess of those accessible in atomistic scale simulations. Given the importance of structure and composition in determining the properties of metallic materials, including nanostructured materials, and the inability of atomistic-scale calculations in predicting the evolution of structure in technologically interesting alloys, it is clear that additional methods are needed for the higher length scales.

The atomistics of diffusion are at the core of many structural evolution phenomena. In multicomponent systems, a full description of diffusion phenomena requires information on system thermodynamics as well as on the local barriers to atomic transport, both for a single solute and in the presence of multiple solutes in concentrated quantities. Great strides have been made in first-principles approaches for calculating hopping barriers in various metallic and intermetallic systems. Connections to continuum properties can be made via the use of cluster expansions combined with kinetic Monte Carlo approaches for the prediction of diffusion at finite temperatures. ${ }^{29,31,32}$ Challenges remain for applying techniques to nondilute multicomponent systems; new algorithms along with increased computer power should permit progress on this necessarily large computational problem. Large-scale calculations could ultimately identify appropriate "averaging" approaches as well as uncover new mechanisms in the presence of high gradients or high concentrations of point or line defects. In fact, Monte Carloinspired approaches are extensively used at coarser length and time scales, e.g., the Potts model for grain growth, sintering, and recrystallization.

One approach that addresses the higher length scale is the phase-field method, where the interface between domains is taken to be diffuse rather than sharp as in the classic approach. The phase-field method employs coarse-grained free energies and 


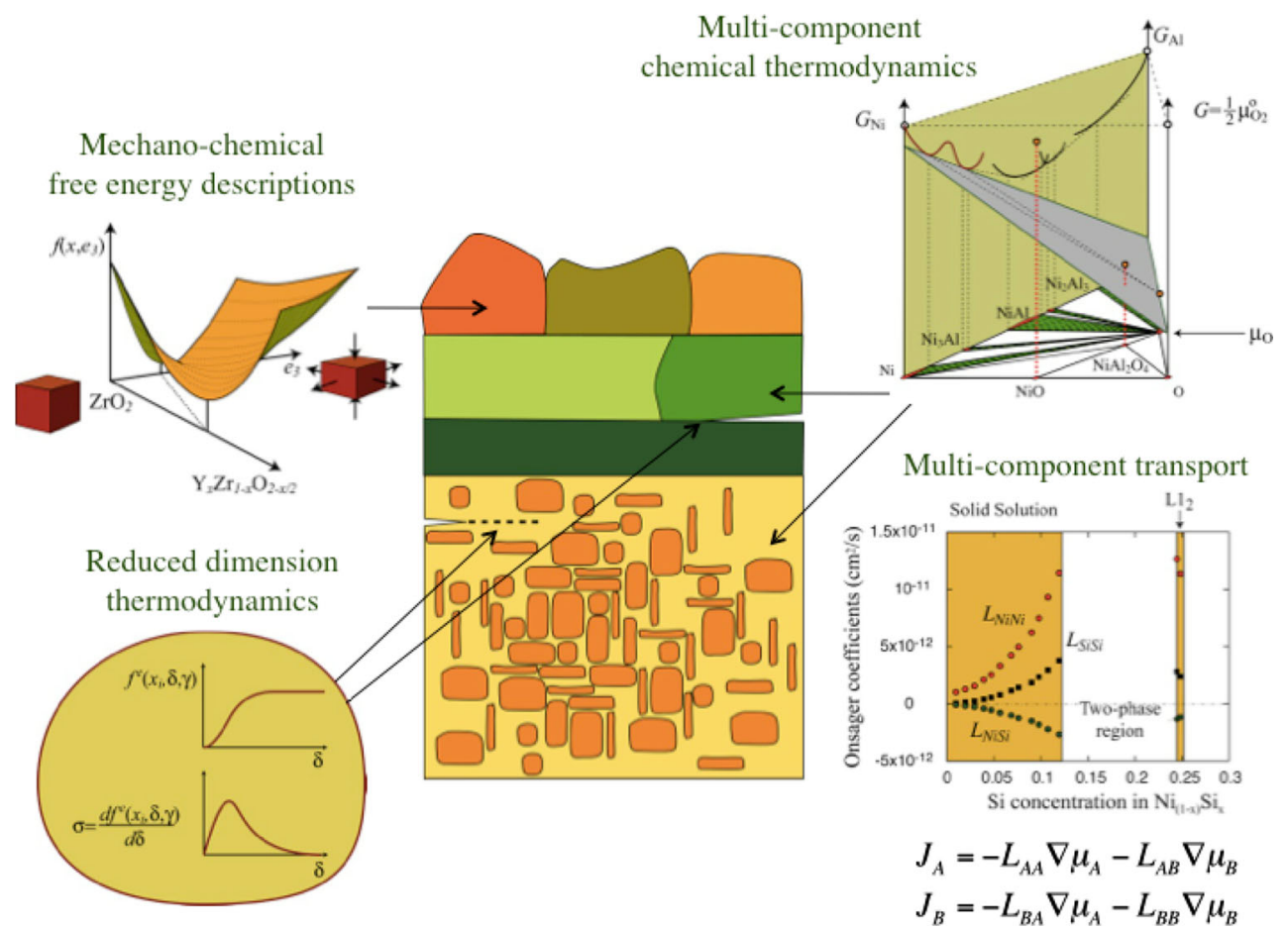

Fig. 2. A computational framework for integration of atomistics, thermodynamics (including stresses), interfaces, and kinetics. ${ }^{33}$

the diffusive dynamics by which microstructure evolves in reality; thus, it can predict the evolution of the structure of a metal on the mesoscale. Because the method is based on free energy functionals, it is possible to add other fields, such as elastic stress and magnetic or electric fields, and to include the effects of the many alloying elements that are typical in technical alloys. Thus, the method provides a bridge between the atomistic and the continuum.

Much work remains to realize the full potential of the approach. The effects of the diffuse interface, usually taken to be much thicker than in reality for computational convenience, remain to be fully understood in many cases. Numerical methods employed to solve these nonlinear diffusion equations need to be developed, with particular attention to those methods that scale well on peta- and exa-scale computers. Although there has been significant progress in developing open-access phase-field codes, e.g., FiPy from NIST, and other codes accessible from platforms such as Matforge (www.matforge.org) or NanoHub, ${ }^{22}$ at this point there is no widely employed standard phase-field code, as there is for density functional theory or molecular dynamics. An important extension of the phase-field method, known as the phase-field crystal method, captures atomic-scale structure through use of an order parameter that represents local density, with the free energy functional minimized by a periodic order parameter configuration. This formulation allows the consideration of grain orientation, interface structure, stresses, and compositional inhomogeneities, but at the longer time scales required for diffusion, permitting semiquantitative scaling of the properties of multicrystalline solidification, dislocation dynamics, fracture, grain boundary energetics (premelting), elastic phonon interactions, grain coarsening, and both linear and nonlinear elasticity and plasticity. ${ }^{34-37}$

While implemented in many different contexts, from a first-principles statistical mechanics point of view, phase-field models can only be considered rigorous when applied to phase transformations and microstructure evolution involving phases that have a group-subgroup relationship, and then only if the coexisting phases are coherent. This includes phase separation between identical crystal structures, differing only in their concentration, as well as order-disorder transformations on a parent crystal structure (e.g., $\mathrm{L} 1_{2}$ ordering within a face-centered cubic [fcc] matrix). Because the coexisting phases are linked by a symmetry group/subgroup relationship, they can transition into each other through a continuous variation of suitably defined order parameters (e.g., composition or order-disorder order parameter). The continuity between the phases allows for the formation of diffuse interfaces separating the coexisting phases. The existence of a symmetry group/subgroup relationship also means that the various phases competing for stability correspond to local minima of a common free energy surface, with interfacial free energies arising from contributions of the homogenous free energy supplemented with a gradient energy correction introduced in the original Cahn-Hilliard-Allen theories. A large number of phase transformations do not 
involve symmetry group/subgroup relationships, but instead they involve phases that are crystallographically very different, necessitating sharp or incoherent interfaces. Because of the incompatibilities in symmetry, the various phases cannot be described by a single free energy surface from which interfacial free energies can be extracted. Instead, the various phases have their own free energy descriptions, and the sharp interfaces separating them will have a corresponding excess free energy. Phase evolution then generally involves diffusion within the individual phases coupled with interface migration driven by chemical potential and stress differences across the interface. The description of these phase transformations with phase-field models can be viewed only as phenomenological, as there is no clear coarse graining procedure available to link the actual crystallographic and atomic-scale processes to the parameters of the phasefield model. More appropriate approaches, although numerically substantially more challenging, are based on sharp interface models, which can be treated, for example, with level set methods.

Rigorous models for structure evolution will ultimately permit tailoring of multicomponent materials, from the mesoscale down to the nanoscale, for optimal properties. For instance, in magnetic alloy systems, nanostructured soft magnetic alloys and nanocomposite permanent magnets rely on the magnetic exchange correlation length as a fundamental length scale. When microstructures in soft ferromagnetic materials consist of nanoscale grains, the resulting coincidence of correlation lengths results in remarkable properties. ${ }^{38}$ However, the preparation of multiphase, multicomponent nanostructured materials for use in bulk form (at higher scale structures) is extremely difficult due to the coarsening driving forces, presenting significant challenges for the control of structure and structure evolution. Alloy and process design, assisted by a modeling infrastructure, could identify multicomponent, multiphase systems that enable revolutionary improvements in materials performance, especially in power-dense conversion and conditioning technologies with greater energy efficiency. Anticipated trends in the performance of magnetic materials, as tracked by the coercivity, that are possible with multicomponent, nanostructured materials are shown in Fig. $3 .^{39}$ Similar discoveries would be anticipated within a vast array of multicomponent materials, including thermoelectric systems based on intermetallics, battery cathode materials, fuel cell membranes and piezoelectrics, multiferroics, hydrogen storage systems, shape-memory alloys, and lightweight, high-temperature structural materials.

\section{The Science Base for Property Prediction Across Length Scales}

The convergence of new structural characterization capabilities, new theory, and vastly improved computational capabilities promises a new era for

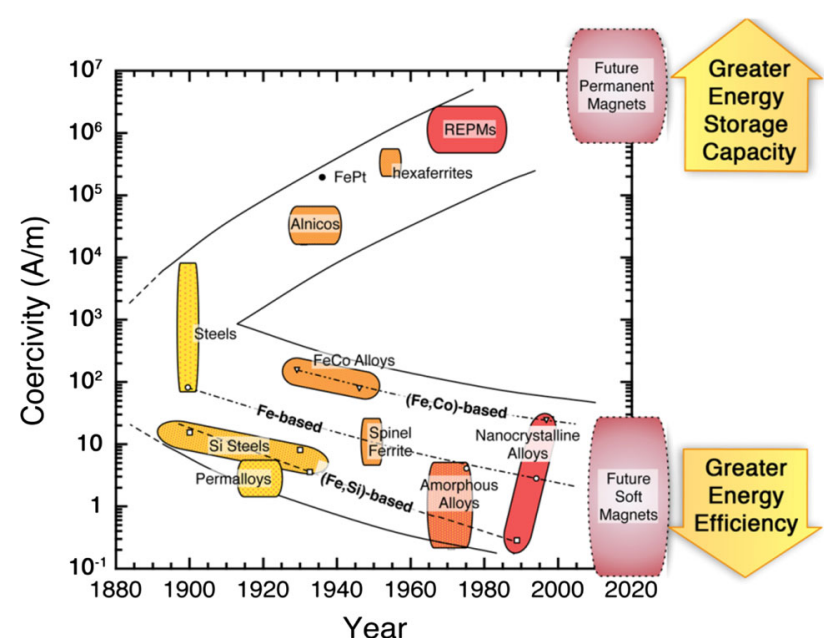

Fig. 3. Timeline of progress in the improved performance for soft and hard magnets as measured by the coercivity of different magnetic materials (adapted from Ref. 39).

material property prediction. A diverse combination of properties is simultaneously needed to optimize deployment of most materials in any engineering system. The properties needed range from melting temperature to fatigue, where the extremes of microstructure dominate the response, as demonstrated in Fig. 4. Although a wide range of properties has traditionally been classified as "structure insensitive," this paradigm fails at specific size scales for particular properties. One well-known example is the decrease in melting temperature with decreasing particle size, i.e., the GibbsThompson effect. In addition to the scale and structure dependence of properties, many properties (such as fatigue) are more sensitive to rare features of material structure than to the "average" structure that would be captured by inspection of random 2-D sections. It is, therefore, important to predict not only "average" properties but also the "minimum" properties and the associated rare, but critical, features that limit design and material performance. It is also important to note that the reliability with which properties can be predicted based on first-principles understanding becomes increasingly difficult as one ascends the length and time scales and the properties become increasingly dependent on rare features.

The new 3-D imaging modalities discussed previously promise the capability of sampling statistically significant volumes of material, capturing features on the extremes of the distribution of structural features and thereby acquiring structural information for the "average" as well as the "minimum" property. New statistical approaches to sampling large data sets, including the statistics of extremes, are needed in conjunction with careful consideration of the representative volume element required for any given property-perhaps best referred to as the "property volume element" (RVE). ${ }^{41,42}$ Such considerations will undoubtedly 


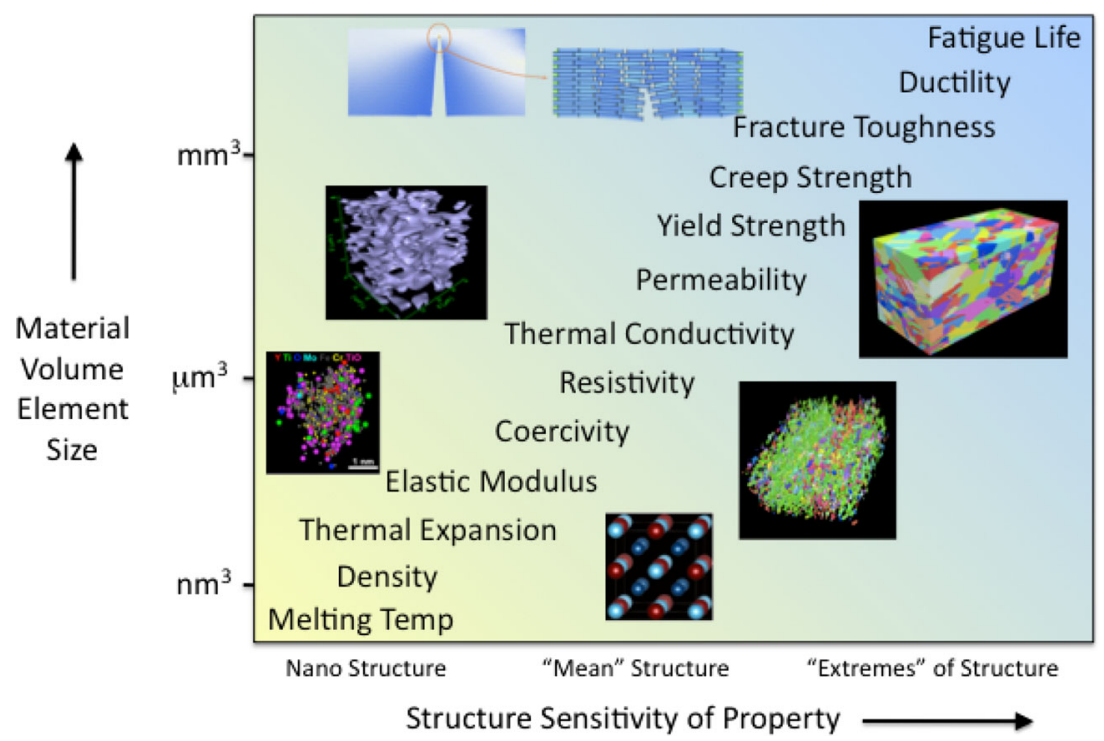

Fig. 4. The volume element of material relevant to the prediction of a spectrum of material properties. ${ }^{40}$

drive the development of new instruments, expand the functionality of existing 3-D characterization approaches, and motivate advances in modeling of composition and structure evolution, as noted in the previous section. A related issue is the level of information that should be retained about 3-D structure for any given material because, in principle, a new design scenario could always change the RVE. Compressive sensing approaches, yet to be applied in any level of detail to materials problems, represent one potentially useful area for further investigation. ${ }^{43}$

Prediction of a significant set of properties for a material requires material information across a wide range of length scales. Connecting information across scales is most often done with "information passing" in which information at a smaller scale is represented so as to reduce the amount and complexity of the information. That reduced representation, which generally is a reduced-order model, is then passed to the next scale. ${ }^{44}$ The use of reduced-order models creates errors in the information passed from lower to higher scales. Since there are few inverse models that go down in scales, the information flows in only one direction, which inhibits our ability to use the current state at the higher scale to help define the information needed from the lower scale. There are many challenges and uncertainties in the "information passing" paradigm. It implicitly assumes that there exist natural coarse-graining lengths at each scale and ignores overlaps of scales, which may not be appropriate in many situations. The reduced-order models add uncertainty when used to describe phenomena at larger scales. ${ }^{44}$ Coarse graining ignores rare and extreme events and structures, limiting their ability to describe such important phenomena as failure. The challenge is that we have no $a$ priori knowledge of which variables at a small scale are critically important at a larger scale. Indeed, the vast majority of variables may have no information value at a larger scale, and it is inefficient to measure or calculate them all. Research is needed to develop new experimental and modeling approaches to identify that critical information.

Metallic materials are pervasive in engineering systems in part due to their ability to undergo plastic deformation without catastrophic failure. The monotonic and cyclic plastic properties of materials (yield strength, hardening rate, creep, and fatigue) are all sensitive to dislocation motion as well as the interaction of dislocations with structural features of the material. Predicting the collective behavior of dislocations and their resultant effect on mechanical properties and failure processes remains a major challenge for metallic materials. ${ }^{45}$ Dislocation phenomena occur over a range of timescales, with fast glide processes that can result in stochastic bursts of dislocation activity $^{46}$ along with slower relaxation phenomena such as dislocation climb. Under cyclic deformation conditions, a fractional portion of the gliding dislocations exhibits irreversible behavior upon reversed loading, resulting in their storage in slip bands that eventually lead to failure. Many aspects of the dynamic behavior of dislocations and the resultant storage and annihilation processes remain to be understood. For example, the role of dislocation sources in the heterogeneity of small-strain plasticity, the dynamics of plasticity in small volumes, and the generation and interaction of dislocations with interfaces are all areas that require more detailed investigation. Given the typical lengths and timescales relevant to dislocation processes, the method of choice for simulations has been one in which the entities of the simulations are not atoms but rather the dislocations themselves, i.e., discrete 
(a)

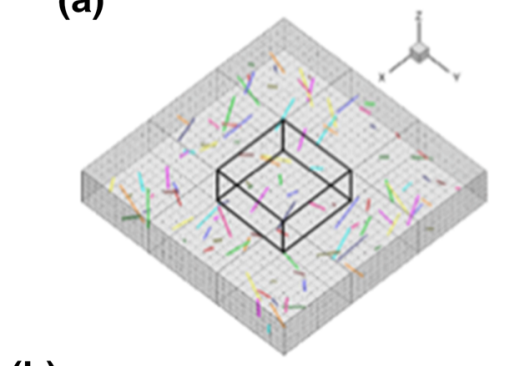

(b)

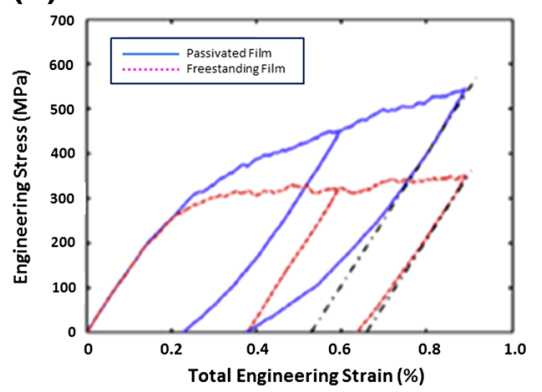

(c) 1

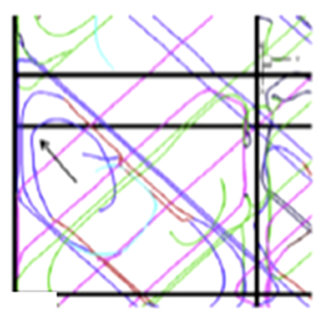

3

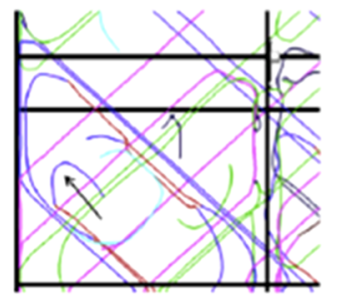

2
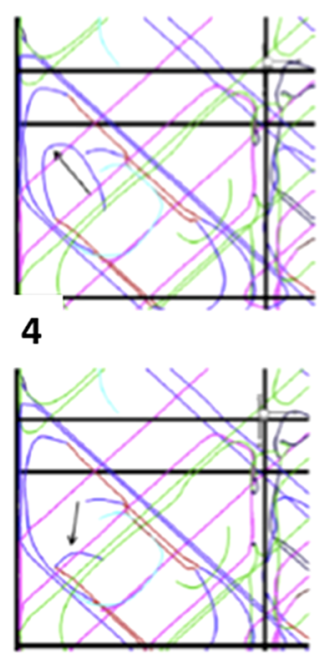

Fig. 5. Discrete dislocation dynamics (DDD) simulations of passivated and free-standing thin films. (a) Schematic of the simulation cell with nine grains, with the center grain shown with thicker lines, BEM mesh with dashed lines, and dislocations as color lines indicating the slip system. (b) Stress-strain curves of freestanding and passivated films during unloading for a system with the grain size and film thickness both equaling $500 \mathrm{~nm}$. (c) Illustration of the reversed motion (panels 1-4) of a pile-up dislocation (marked with an arrow) in a passivated film during unloading. ${ }^{65}$

dislocation dynamics (DDD). The computational framework for DDD has reached a level of maturity for some problems, primarily where the plastically deforming volumes are highly constrained, such as in micropillars ${ }^{47-56}$ and thin films ${ }^{57-65}$ (Fig. 5).

Major challenges remain for DDD simulations of bulk plastic deformation processes. Dislocation dynamics simulations include all atomistic-dislocation core-level events, including dislocation reactions, such as annihilation and junction formation, as well as all activated processes, such as processes that have an energy barrier and are thus thermally activated. In the latter category are the well-known processes of cross slip and climb. The classic models implemented to date, for example the Escaig model of cross slip, ${ }^{66-68}$ do not capture the complexity of dislocation substructure evolution, suggesting that further investigations of these fundamental aspects of plastic deformation are needed. Notwithstanding the insights into dislocation interactions that lead to work hardening, there is no means of generalizing the results of DDD simulations to obtain quantitative descriptions of work hardening for polycrystals. Recent atomistic simulations have provided new insights and directions for development of this modeling approach. ${ }^{69,70}$ It is anticipated that DDD simulations will provide insights to small strain and high-rate deformation, ultimately guiding the development of macroscale constitutive models (for example, crystal plasticity models) for monotonic and cyclic deformation. Beyond this, theory that can guide the prediction of other statistical aspects of deformation, for example, the tendency toward avalanche behavior, ${ }^{46}$ remains to be incorporated into property prediction frameworks. At the mesoscale, plasticity is still challenged by an incomplete understanding of slip transmission across interfaces and boundaries. One promising approach in quantifying slip transmission and the role of interfaces and grain boundaries in localizing strain is in combining molecular dynamics simulations and measurements of a large set of grain boundaries, with a range of incident slip types and orientations, as shown in Fig. 6. ${ }^{71,72}$ The simulated energy barriers for slip transmission have direct implications to modeling crystal plasticity, fatigue, fracture and creep at the mesoscale for specific metal structures. Crystal plasticity approaches are effective for predicting the evolution of texture at large strains in fcc metals ${ }^{73}$ but are less effective at predicting small-strain behavior on a grain-by-grain basis. ${ }^{74,75}$ The degree to which low strain behavior is influenced by factors such as elastic anisotropy, the distribution of dislocation sources, and 3-D microstructural "neighborhoods" (that in the limit could be characterized via $n$-point statistics) remains to be investigated in more detail, with close linkage of experiment and theory needed.

Functional materials including thermoelectrics, magnetic materials, shape-memory alloys, catalysts, and electrodes for fuel cells and batteries could greatly benefit from research aimed at fundamental properties underlying their function. As computational power continues to improve, the ability to calculate the properties of more complex, multicomponent materials within the framework of density functional theory improves. However, the temperature dependence of properties and the kinetic phenomena that intervene in these systems are areas that remain in need of fundamental research. Also, as discussed in more detail 
(a)

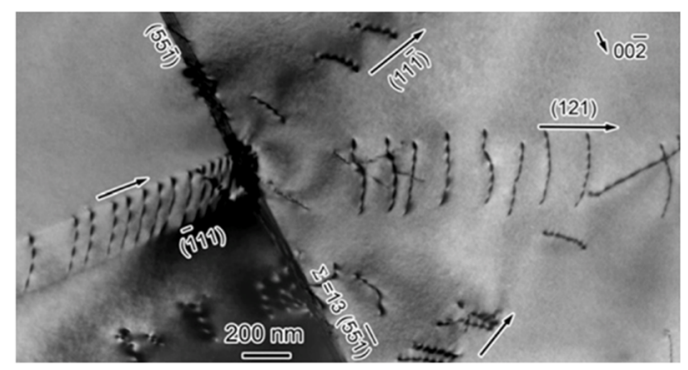

(c)

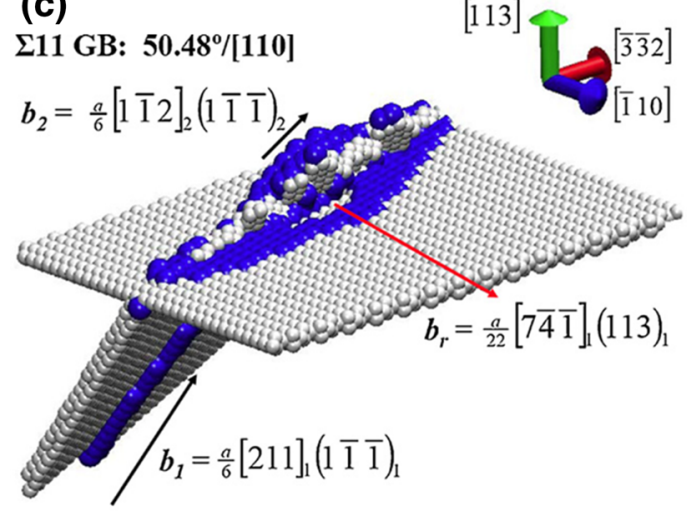

(b)

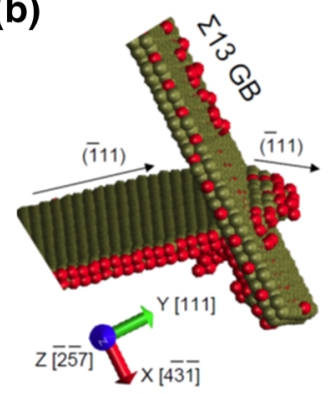

(d)

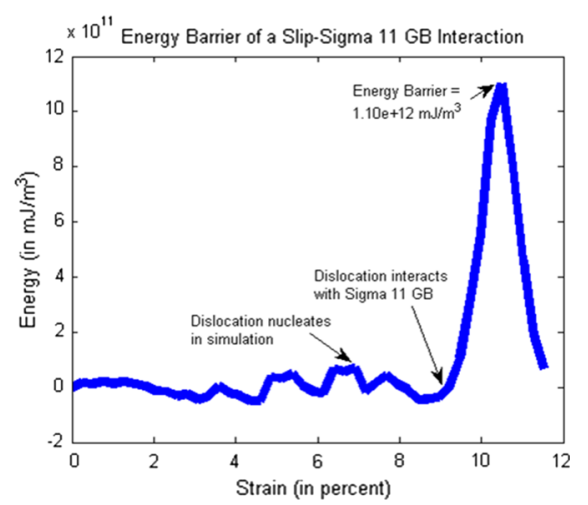

Fig. 6. (a) In situ TEM micrograph of slip transmission in a low stacking fault energy material for a $\Sigma 13$ grain boundary (GB). (Micrograph is a composite figure formed from images in which the diffraction condition was specific to each grain) molecular dynamics (MD) simulations of slip transmission through a $\Sigma 13 \mathrm{~GB}$ (b) compare well with the experimental results. (c, d) Interactions of dislocations with $\Sigma 11$ GB with the resulting dislocation reactions and the corresponding energy barrier calculations for slip to penetrate the $\Sigma 11 \mathrm{~GB} .{ }^{71,72}$

below, the ability to model interfaces and their effect on properties remains a major fundamental barrier to scientific progress, particularly for functional materials, where applications often demand submicrometerscale layering or percolated structures with specific geometries for optimal properties.

With respect to the translation of new materials into engineering applications, success in property modeling and prediction translates to dramatically reduced amounts of testing, eliminating the need for expensive and time-consuming "design of experiments" approaches that are currently practiced widely in industry. ${ }^{76}$ Testing will instead be driven by the needs for model development and for verification and validation of these models, for bulk materials as well as for nanoscale structures. This, in turn, is likely to motivate new site specific, in situ and/or rapid testing techniques for the spectrum of metallic materials and properties of interest.

\section{Local Phenomena at Interfaces-Experiment, Theory, and Simulation}

Recent research on the structure and stability of metal surfaces, interfaces, and defects, as well as their motion and interaction with the environment, have strong implications for our understanding and control of atomic-scale and molecular-scale reactions and for creating new materials with dramatically improved functionalities and properties. Metals are often used in contact with aqueous solutions and other liquids-in biological environments, in many electrochemical energy technologies, and in structural applications. In some cases, electrochemical potentials are applied or generated across these interfaces, but these simply add to the generally complex chemistries ubiquitously in play. In structural applications, these potentials manifest themselves as corrosion, especially in lightweight structural alloys containing high percentages of reactive metals. For energy conversion in batteries, fuel cells, and capacitors, they enable the charging and discharging chemistries and processes, which often involve dissolution and redeposition of a metal. In a variety of distinct phenomena, they are connected generally by a common structure of a metal/electrolyte interface whose local properties control the aggregate response of the material. Throughout the workshop, the science that underlies these developments was discussed.

Understanding the interaction of metal surfaces and interfaces with fluid environments is clearly a challenge in many research areas and for many fundamental processes. For example, the combination of chemistry, electrochemistry, crystallography, and mechanical properties in determination of the performance of metal surfaces used as electrodes for 
batteries or fuel cells, and even catalysts, is complex. Although the essential interface processes are the same at surfaces and interfaces at all size scales, from bulk materials to nanoparticles and nanoporous films, the atomic-scale features and configurations that exist on highly curved or chemically heterogeneous surfaces have been found to profoundly influence their activity with the environment. Charge transfer, the growth of passivating or poisoning layers, and surface reconstruction also have significant impact on the performance, stability, and reliability of materials. As a result of the growing ability to track atomic and electron transport at and along surfaces, as well as their local surface structures and strains, it is becoming possible to design surfaces at the nanoscale with specific features, such as kinks and steps, and to stabilize such structures through controlled segregation and composition control.

One example illustrating the potential of this holistic approach at the nanoscale is in the atomic origins of the high catalytic activity of nanoporous gold. ${ }^{77-80}$ In these studies, measurements of the 3-D surface structure, composition, and strain of nanoporous gold revealed (I) the likely mechanisms for the unexpectedly high catalytic activity of nanoparticle $\mathrm{Au}$ and nanoporous $\mathrm{Au}$ in $\mathrm{CO}$ oxidation reactions and (II) a processing route that creates these interesting metallic nanostructures in a controlled manner. Nanoporous gold is synthesized as self-supporting films by dealloying $\mathrm{Au}-\mathrm{Ag}$ films, a process strongly related to the corrosion of metallic alloys. The resulting bicontinuous 3-D nanostructures have surfaces with positive and negative curvatures, with large curvature gradients between them (Fig. 7). The catalytic activity of nanoporous gold is associated with its structure at the nanometer scale, specifically the density of geometrically necessary kinks, steps required to transition from positive to negative curvatures and associated local surface strains. Residual segregated Ag provides stabilization and suppression of (111) faceting for these structures. Going beyond the traditionally planar view of interfaces into a structurally and compositionally rich $3-\mathrm{D}$ view at the nanoscale will provide not only a more accurate picture of the underlying thermodynamics and kinetic processes, but also greater prediction and control of interfaces for future applications.

Some important aspects of the fundamental science of metal/electrolyte interfaces, for example, include (I) surface mobility of metal atoms along the interface, ${ }^{81,82}$ (II) dissolution of the surface, ${ }^{83}$ (III) deposition on the surface, ${ }^{84-86}$ and (IV) reaction chemistry. ${ }^{87,88}$ Such fundamental physical phenomena inform slightly higher-level phenomena such as nucleation and growth of nanoparticles, ${ }^{89-91}$ morphological stability, ${ }^{92,93}$ and corrosion. ${ }^{94}$ Modeling methods, such as first-principles models of the metal/electrolyte interface ${ }^{95}$ and characterization tools, such as in situ TEM, ${ }^{96}$ are in their infancy.
A challenge not limited to nanoscale processes at metal surfaces is to design experiments and models that can be coupled along time and length scales. Development of the theory-simulation-experimental linkages that can reveal the mechanisms of metal interface reactivity, stability, performance, and reliability is a scientific grand challenge in this area. Such advances are needed for application to the broad range of metals and environments noted above. For example, while many examples exist of the extension of a model to attempt to account for bulk electrical performance of a battery, the use of general metal-electrode interface parameters suggests rightly so that the actual processes are still unknown in many cases.

Similarly, the need for high-speed characterization methods to match the time scales of diffusion calls for further advances in ultra-fast microscopy, as well as the development of more "bulk" techniques. Matching the size scale of interface reaction experiments, such as through scanning probe techniques and environmental transmission electron microscopy (ETEM), to the time scales of molecular dynamics, phase-field, and phase-field crystal simulations for a material and its surrounding environment will be critical to determining the mechanisms that control performance and long-term stability of materials.

Another related, but distinct, area of importance is in matching the characterization environment with the relevant experimental and application environments. With the increasing use of atom probe tomography, atomic resolution TEM, and ETEM, it is becoming clear that the structures so often captured in ultra-high vacuum (UHV) or "dry" environments may not be the actual structures present during interactions with "dirty" real environments in other laboratory experiments or in use. Surface reorganization and reconstruction, adsorption and dissolution, and metastable phase formation are all processes that change interfaces. The continuing development of new techniques that can deal with increasingly complex environments will enrich both the underlying scientific base as well as the ability to create and field new metals, metal nanostructures, and technologies derived therefrom. One such innovative, even futuristic, technology described at the Workshop was electrochemically self-propelled multisegmented nanowire motors and swarming nanoparticles envisioned for biomedical applications.

\section{Prediction and Control of the Morphology, Microstructure, and Mechanical Properties of "Bulk" Nanostructured Metals}

"Bulk" nanostructured metals, that is, metals that can be used for structural applications but possess a structure that is controlled at or near the nanoscale, will be central in a variety of applications related to the subject areas of energy, environment, and sustainability. Nanoscale research is, therefore, 

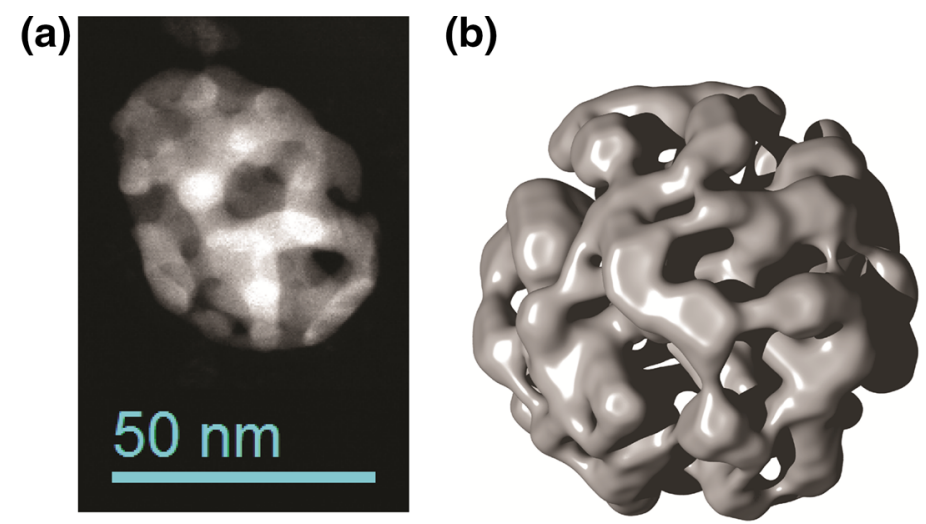

(c)
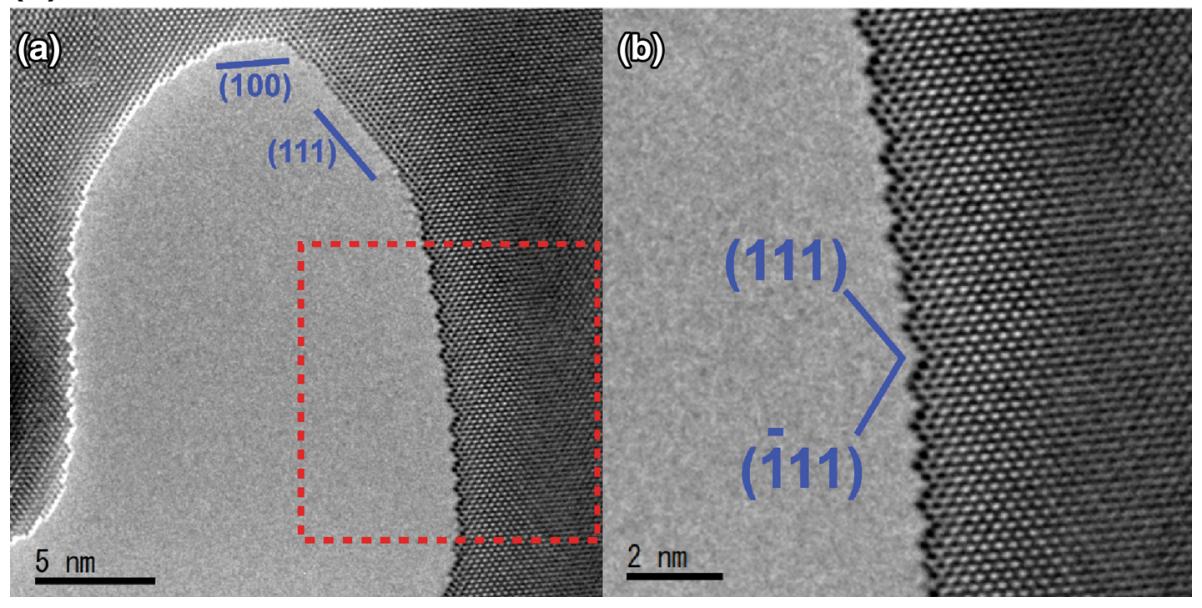

Fig. 7. Different synthetic, computational, and characterization tools applied to the study of "nanoporous gold," made by electrochemical dealloying. (a) TEM showing dissolution of silver from silver/gold nanoparticles yielding nanoporous nanoparticles that exhibit unusual nanoscalerelated kinetics and morphological evolution ${ }^{77}$ (b) Large-scale atomistic kinetic Monte Carlo simulations combined with novel image processing methods leading to a theoretical understanding of these materials. ${ }^{78}$ (c) Atomic resolution environmental transmission electron microscopy (ETEM) of nanoporous gold revealing origin of its catalytic activity: Here, the presence of a CO background pressure is shown to induce a faceting reconstruction in high-gold-content materials, suppressing $\mathrm{CO}$ oxidation. ${ }^{80}$

not just limited to the creation and behavior of individual nanoparticles or nanostructures. It must include the formation and resulting properties of nanoscale to mesoscale assemblies of nanostructured materials. Examples of this length scale linkage include the following:

- The yield criteria for nanostructured metals and thin films, their damage evolution, multiaxial stress state effects, strain-rate dependence, and their stability and behavior as a function of temperature

- The role of environment in plastic response of nanostructured metals

- The failure and fatigue of nanostructured metals and thin films, and their durability in extreme environments

- The ability to fabricate precise "bulk" nanostructured materials in 3-D, especially with multiple components, phases, and classes of materials

Although there are many applications of such research, two are highlighted here. One possible application is in radiation-tolerant metals needed for next-generation nuclear power generation, and ultimately for fusion reactors. Strategies for success in this area include fabrication of metals with both adequate strength and a continuing ability over time to absorb point defects formed by radiation, both properties potentially aided by nanoscale precipitates, grains, and/or nanolaminate configurations. ${ }^{97,98} \mathrm{~A}$ second application is in new magnetic nanoscale materials that can serve as rare-earth magnet substitutes. ${ }^{99}$

Developing and maintaining nanostructure features in bulk materials is a very rich area of fundamental metals research, including the following:

- Laboratory synthesis of bulk nanostructured metals,

- Stability under different driving forces,

- Characterization of bulk nanostructured materials at the needed spatial and temporal resolution without relaxing the physical constraints needed to preserve their "bulk" character, and 
(a)

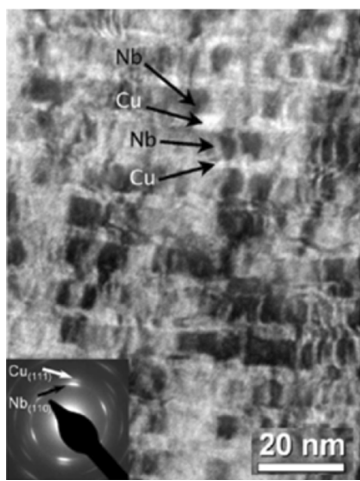

(b)

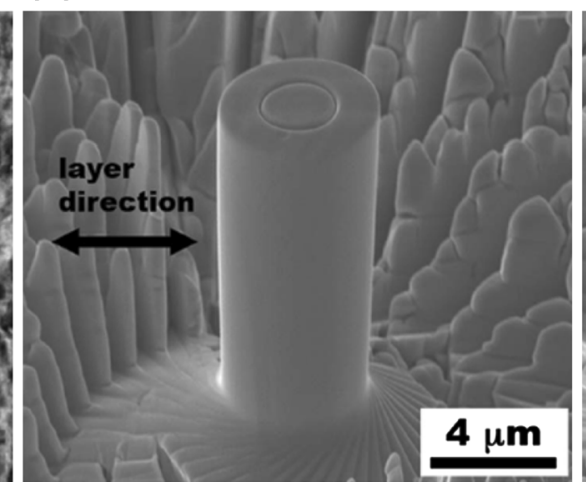

(c)

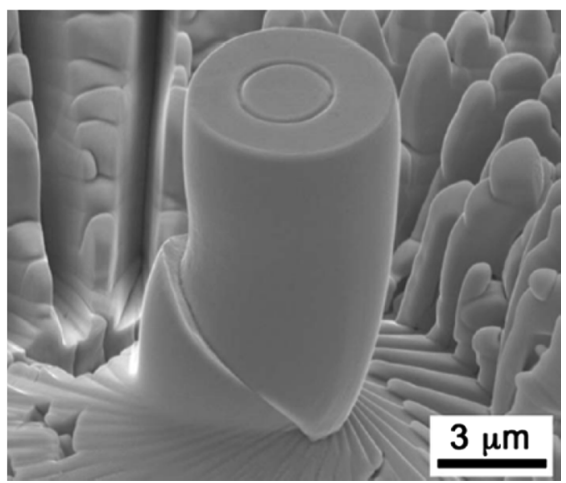

Fig. 8. TEM bright-field micrograph of as-deposited $40 \mathrm{~nm} \mathrm{Cu} / 40 \mathrm{~nm} \mathrm{Nb}$ nanolaminate material selected-area diffraction pattern showing $\{111\} \mathrm{Cu} \| 110\{\mathrm{Nb}\}$ interface texture (a), an as-fabricated micropillar (b), and a micropillar that underwent $25 \%$ compression before failing by catastrophic shear (c). ${ }^{104}$

- Theory and computational methods that can help inform and even drive the experimental work.

Fundamental knowledge regarding the structure dependence of surface and interfacial energies, phase diagrams, and interface mobilities is lacking, as is quantitative information on diffusion in the bulk, on surfaces, and along grain boundaries. These factors ultimately determine the microstructures that can be obtained starting from nanoscale features. For example, a model for the stability of binary nanocrystalline alloys with respect to grain growth and phase separation was recently developed and used to construct "nanostructure stability maps," with several new types of stable nanostructures identified. ${ }^{100,101}$ Likewise, molecular dynamics simulations of nanocrystalline, multicomponent alloys using angular-dependent interatomic potentials, such as in the $\mathrm{Cu}-\mathrm{Ta}$ system, are revealing segregation and nanoclustering effects that pin grain boundaries, prevent grain growth, and thereby stabilize nanostructures and create stronger materials than if the alloying agents were uniformly distributed. ${ }^{102,103}$ Experimental examination of these models' predictions will likely reveal heretofore unidentified effects of composition and structure on the stability of nanocrystalline materials. With research targets such as those noted here, research on "bulk" nanostructured materials fits well within the MGI and NNI goals. Ultimately for translation into applications, the linkage between structure and engineering properties (e.g., constitutive relations) is also needed, in addition to the more fundamental chemical and physical linkages between synthesis and structure.

Three central research directions have been identified for "bulk" nanostructured materials. First, new techniques are being developed to characterize materials at unprecedented levels of detail, such as x-ray and TEM tomography, the many variations of serial sectioning, and high-speed in situ microscopy. Such characterization is yielding new information about grain orientation, stress states, strength-hardening-precipitate locations, and dislocation distributions, all with the needed spatial and temporal resolution. For progress to continue, particularly for materials mechanics research, the data and modeling challenges described above in 3-D and 4-D materials science must be resolved. Second, measurements of the properties of nanostructured materials necessitate new fabrication approaches, as exemplified by techniques such as FIB milling of micropillars that are subsequently compression tested and imaged in situ within the scanning electron microscope (Fig. 8). ${ }^{104}$ Third, useful scientific knowledge will be found only if the interpretation of such experiments rests on a firm foundation of mechanics theory, much of which is lacking for nanostructured materials.

\section{Functionality and Control of Materials Far from Equilibrium}

The ability to predict and control local driving forces for diffusion and interface motion in systems far from equilibrium permits the design and creation of structures at the atomic, nanometer, and micrometer scales. By applying such control, new fabrication pathways are providing access to novel glass structures, nanoparticles, nanowires, and thin films with equally novel properties, functionalities, and applications. For example, the understanding of atomic configurations in the glassy state and crystallization kinetics as a function of cooling rate have advanced the discovery of large sets of rare-earth containing and Fe-rich bulk metallic glasses with unique mechanical, magnetic, and corrosion properties. In another example, nanoscale core-shell precipitates formed by a two-step heat treatment, whereby the core formed at high temperature provides a template for growth of the shell at lower temperature (Fig. 9a), represents the promise of nonequilibrium paths for improving material properties. For the example of AlLiSc core-shell 
(a)
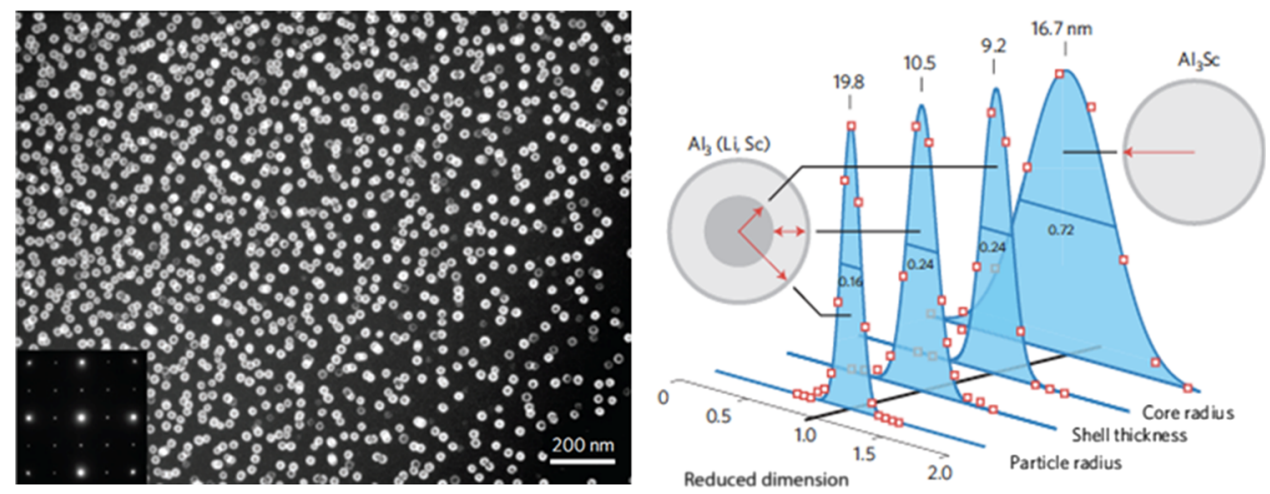

(b)

(c)
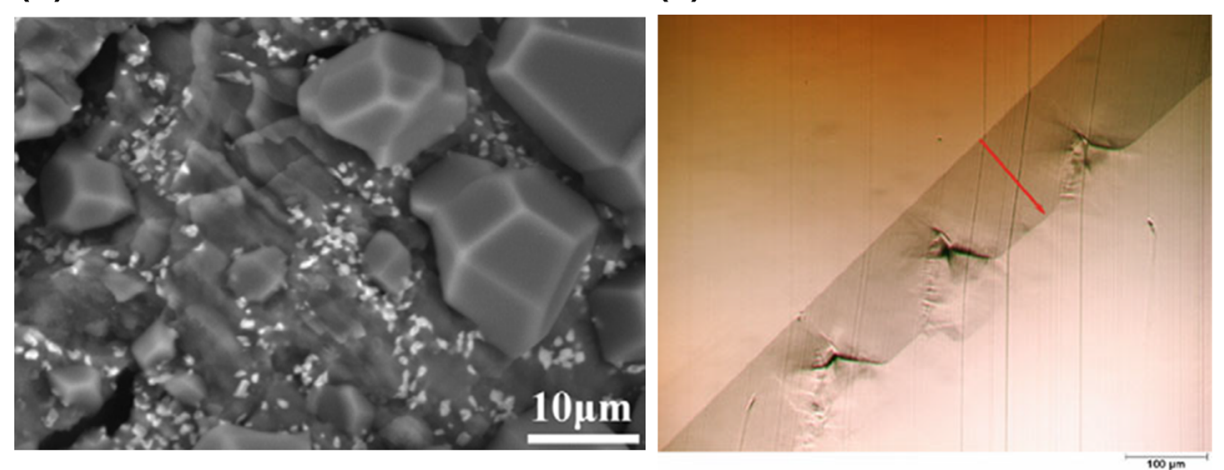

Fig. 9. (a) TEM dark-field micrograph (left) showing a highly uniform distribution of core-shell AlLiSc precipitates with $L 1_{2}$ structure. The selected-area diffraction pattern inset shows strong fcc reflections and weaker $L 1_{2}$ superlattice reflections in a square pattern typical of an [001] crystal orientation. Particle size distributions (right) narrower in core, shell, and core-shell sizes in the present alloy are much narrower than in typical AISc binary alloy particles. ${ }^{105}$ (b) SEM images of large crystal formation after the first hydrogenation cycle of a highly deformed Mg-Ni alloy. ${ }^{106}$ (c) Optical micrograph of an Al bicrystal showing the coupling between shear and grain boundary migration and the generation and growth of new grains during migration of $76.3^{\circ}\langle 100\rangle$ tilt grain boundary after annealing at $370^{\circ} \mathrm{C}$ for 60 min under a tensile stress of $0.84 \mathrm{MPa} .^{107}$

precipitates, this structure leads to an improved resistance in irradiation environments. ${ }^{105}$ Exploiting the full potential of materials far from equilibrium will require an improved understanding of phase and structure stability, nucleation, and the thermodynamics and kinetics of diffusion and interface motion at surfaces, highly curved interfaces, and complex grain boundaries. Additional examples are presented here that illustrate the possible impact of research in this area on both scientific understanding and applications of the resulting nanostructures and thin films.

Nearly defect-free, high-aspect-ratio metal nanowires have been fabricated through low supersaturation growth (MBE) on oxidized and unoxidized silicon single crystals partially coated with carbon. ${ }^{108}$ The facetted nanowires were observed to grow predominantly from the metal/substrate interface (from the base of the nanowire), with nanowire nucleation occurring where the substrate was not covered by carbon. This technique does not require patterning of the substrate, i.e., construction of channels for deposition that must subsequently be removed to leave free-standing nanowires, or the presence of catalyst particles such as in vapor-liquid-solid (VLS) or vapor-solid-solid (VSS) growth. Furthermore, the structural evolution during the nanowire formation process must be related, in an inverse way, to grain growth and dewetting of polycrystalline thin films on substrates. ${ }^{109,110}$

Complex pattern formation during dewetting of epitaxial single-crystal metal films on substrates (Ni on $\mathrm{MgO}^{111,112}$ and $\mathrm{Au} / \mathrm{Fe}$ on sapphire ${ }^{113}$ ) is beginning to reveal both the competitive processes involved in dewetting, grain growth, and thermal stress relaxation, and the control in pattern formation achievable from the nanoscale through the mesoscale. ${ }^{114}$ Crystallographic effects, as well as hole nucleation from surface defects, are key in destabilizing thin-film systems. The formation of large facetted crystals during grain growth and dewetting of thin films during hydrogenation of $\mathrm{Mg}$ also suggests the importance of stress-induced coarsening to thin-film stability in the presence of a phase change. ${ }^{106}$

Research on modeling and measuring stress generation and relaxation in thin films by competing processes during thin-film formation, ${ }^{115}$ thermal cycling, ${ }^{116}$ applied stresses, ${ }^{107,117}$ or isothermal stress generation processes, such as intermetallic formation during room-temperature annealing of $\mathrm{Sn}$ 
(a)

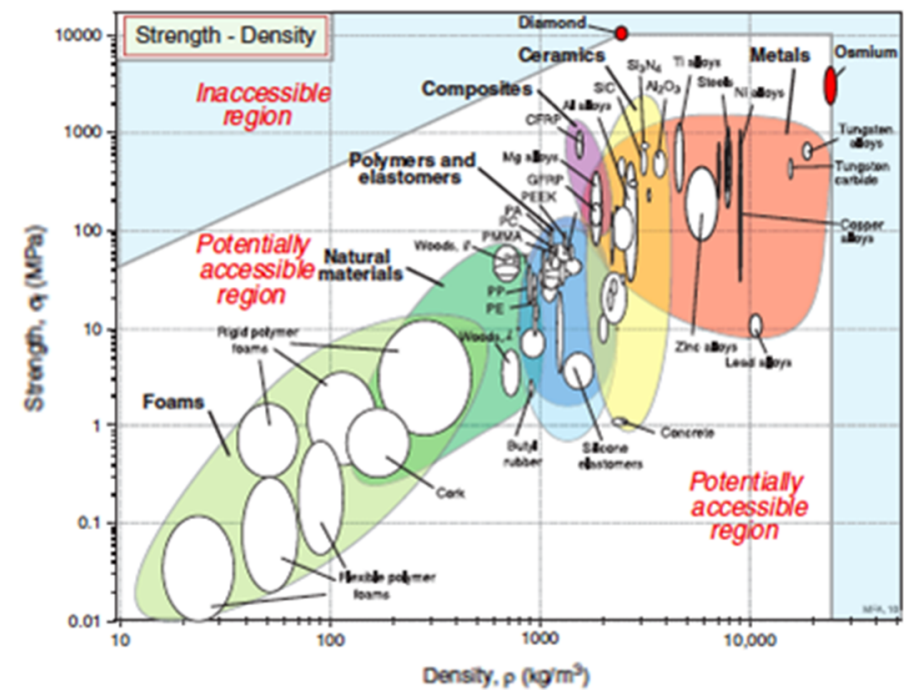

(b)

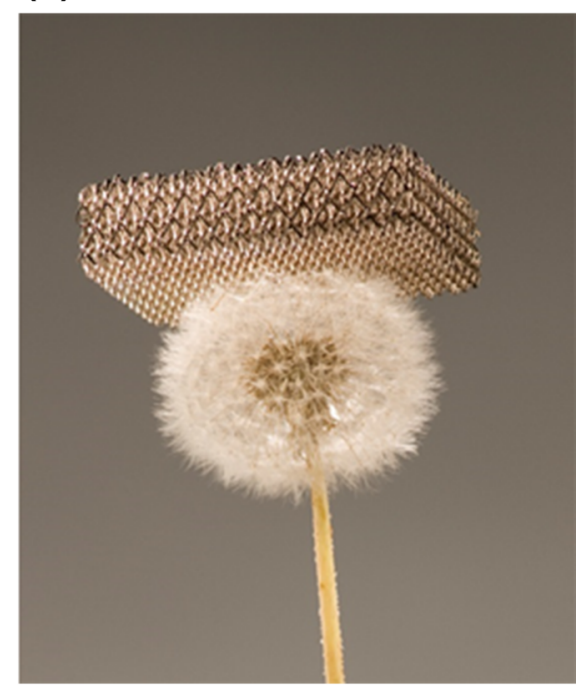

Fig. 10. (a) Potentially accessible property space that can be accessed by hybrid structures, adapted from Ref. 122, and (b) an ultralight weight metallic truss structure supported by a dandelion (approx. $4 \mathrm{~cm}$ diameter ${ }^{125}$ ).

films on $\mathrm{Cu},{ }^{118-121}$ is revealing the role of microstructural heterogeneity and crystalline anisotropy on the response of different thin films to stress. Further progress will require a more detailed understanding of how grain boundaries and interfaces migrate and respond to applied stresses, the mechanisms and conditions for coupling stress and grain boundary motion, and the ability of grain boundaries and surfaces to act as vacancy sources and sinks for diffusional creep. ${ }^{117,118}$ In the future, this research will be important to producing stable, high-performance alloy film applications as diverse as solar cells and microelectronics, to catalysts, to corrosion-protection and thermal barrier layers.

\section{Hybrid and Multifunctional Materials Assemblies}

Hybrid combinations of metals with other materials classes assembled into designed structural elements such as Kagome lattices, truss or honeycomb structures, sandwich panels, foams, or zero or negative thermal expansion structures have the potential to expand property space, filling voids that are naturally present with monolithic materials. An example of the expansion of potentially accessible properties in strength-density space by Ashby is shown in Fig. 10a. ${ }^{122}$ Using hybrid structures to expand the design space for other combinations of properties could result in a vast array of new systems with integrated electrical, thermal, optical, and/or mechanical functionalities. ${ }^{123,124}$ An example of a nickel truss structure with a density of approximately $10 \mathrm{mg} / \mathrm{cm}^{3}$ is shown in Fig. 10b. This structure, fabricated by a self-propagating photopolymer waveguide technique followed by nickel electrodeposition, can recover from $>50 \%$ compression and demonstrates large energy absorption under cyclic loading conditions. Folding and morphing structures constructed from metallic systems potentially offer for additional dimensions of control and functionality in sensors and microsystems, as well as in larger structures. ${ }^{124}$

Although the promise of hybrid multifunctional systems is immense, there are wide-ranging technical challenges yet to be addressed. First among these is the problem of structural and topological optimization that jointly considers the constraints of synthesis processes. For example, a complex distribution of two sets of materials may optimize a property, but the resolution of a $3-\mathrm{D}$ printing process may not be sufficient. There are many gaps in the basic understanding of the additive, digital, and 3-D manufacturing processes that will ultimately enable this class of materials. The physical processes that permit layer-by-layer deposition in general are still not understood well enough to select processing parameters or predict final properties. A basic understanding of the energy deposition processes and material response to electron and laser beams is needed for progress in processing across a wide range of materials systems. Beyond this, selective fusion of powder particles, sintering, photopolymerization, local melting within packed powder arrays, and the flow of viscous binder-powder mixtures all require further study. Given that melting and sintering are often embodied in the processing, residual stresses also become a major consideration; improved process models based on sound metals science are needed to address these issues.

\section{Materials Discovery and Design: Enhancing the Theory-Simulation-Experiment Loop}

The computational resources that have recently become widely available to researchers are 

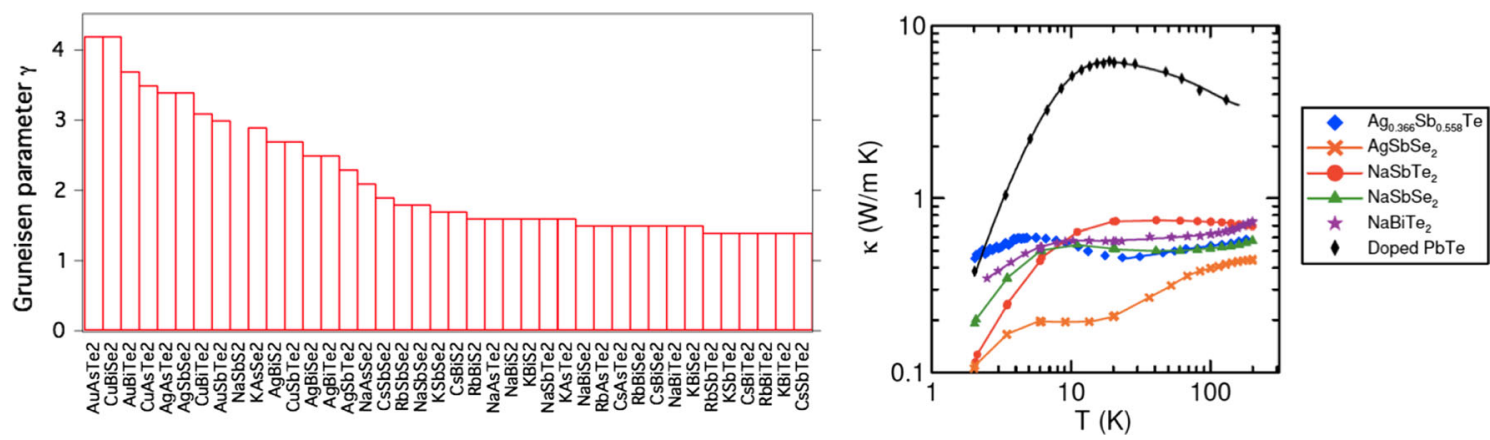

Fig. 11. Systematic ab initio calculations for screening of systems for new thermoelectrics and the results of experiments on the most promising candidates (adapted from Ref. 127).

changing the landscape for materials discovery and design. Atomistic calculations with density functional theory can now efficiently guide the search for multicomponent materials with desirable electronic, thermal, elastic, or thermodynamic properties. ${ }^{126,127}$ An example highlighted at the workshop was centered on thermoelectric materials based on $\mathrm{PbTe}$, which currently exhibits among the highest values of the $Z T$ figure of merit. While it is known that greater additions of solute can further improve thermoelectric properties, the potential experimental search space is large, and it is difficult to experimentally isolate the effects of solute on thermal conductivity compared with electrical conductivity. Starting with PbTe, band structures and the Grueneisen parameters were quickly calculated for $72 \mathrm{ABX}_{2}$ compounds, enabling a systematic selection of compositions for experimental synthesis and thermal conductivity measurements, as demonstrated in Fig. 11. ${ }^{127}$ Fabrication of high quality experimental materials for screening purposes is extraordinarily time consuming, and computational tools can enable a feedback loop that greatly reduces the time required to search for promising new materials. $^{76}$

Because materials must typically exhibit a suite of favorable properties, rather than a single favorable property, rigorous models are needed to predict properties sensitive to material structure across all the length scales demonstrated in Fig. 4. As discussed earlier, the availability of $3-\mathrm{D}$ and 4-D structural data will be an essential element of progress on this front. Protocols for passing only essential structural information from one length scale to the next and one property model to the next are needed. Where models for material behavior are not yet predictive, a close combination of experiment, theory, and modeling can greatly accelerate their development and validation. Additionally, where physical models are lacking, informatics tools are needed to extract information from the vast array of materials literature and to develop correlations among processing, structures, and response at and across all pertinent scales. Accelerating materials discovery and development will require not only computational tools but also experimental tools for property measurements that can efficiently and rapidly quantify properties at the appropriate length scales. For example, as nanostructured materials emerge, some properties need to be measured at the nanometer scale. An example from the workshop of a micro-electro-mechanical systems (MEMS)-scale device developed for testing nanowhiskers is shown in Fig. $12 .{ }^{128}$ New, high-throughput rapid-characterization techniques for a variety of transport properties have also recently emerged. ${ }^{129,130}$ Expanded, rapid approaches for the site-specific measurement of a broader array of properties could dramatically expand the search space for new metallic and nanostructured materials. Finally, it is recognized that an efficient theory-computation-experiment feedback loop will typically require a pairwise interaction of researchers, at a minimum. Resources to support groups of two or three investigators would strongly enhance this approach.

\section{MATERIALS RESEARCH INFRASTRUCTURE}

Critical to continued advances in materials research is the availability of a wide spectrum of advanced instrumentation. As the complexity and the spatial, temporal, and chemical sensitivities of materials research instruments continue to increase, the cost of acquiring, maintaining, and using these instruments also increases. The stateof-the-art transmission electron microscope is an example of an instrument that has traditionally existed within the individual infrastructures of nearly all materials research institutions. However, the most recent generation of aberration-corrected transmission electron microscopes are priced well beyond the resources of most individual institutions and require professional staffing for their optimum use. Furthermore, suites of instruments, rather than a single instrument, are required to tackle emerging materials research problems. For example, 3-D atom-probe tomography usually requires not only an atom probe instrument but also a focused-ion beam microscope for sample preparation and a TEM for characterization of the resulting 

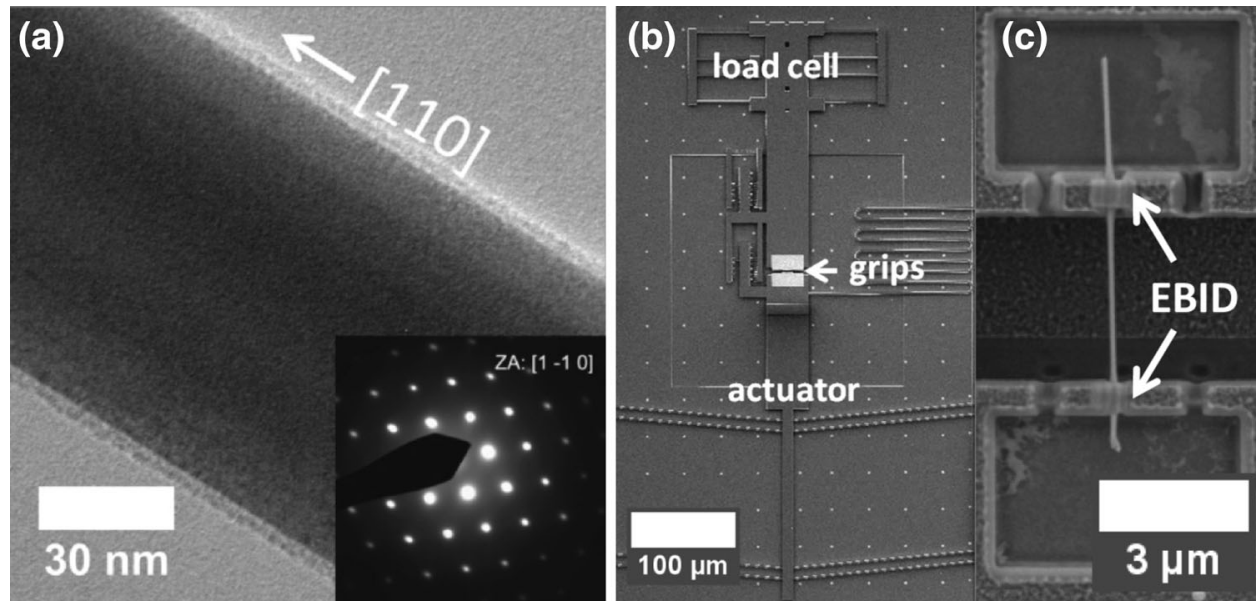

Fig. 12. Small-scale testing of Pd nanowhiskers: (a) a TEM image and SAED diffraction pattern (inset) for a Pd specimen and (b) a thermally actuated tensile testing stage on which specimens are tested. The comb features to the side of the grips may be used for tracking displacements of the load cell and actuator. (c) Pd NW across the testing grips. ${ }^{128}$

samples. As the NSF and the Department of Defense (DOD) are the sources of federal resources for instrumentation for U.S. universities, a strategic, long-term materials instrumentation plan with substantial community input is essential. The Major Research Instrumentation (MRI) Program plays an essential and critical role for developing and acquiring new materials instrumentation, as does the DOD Defense University Research Instrumentation Program (DURIP). However, the NSF programs that support smaller-scale instrumentation have been scaled back; an example of this is the now inactive Instrumentation for Material Research program. Although the Materials Research Science and Engineering Centers (MRSEC) program commits significant resources to instrumentation and thus provides major benefits to MRSEC institutions, it has limited impact on the general materials community. Unquestionably, the availability of more grant opportunities for small to intermediate scale instrumentation would have a strong, positive impact on the materials community, particularly on junior researchers who are in the process of building laboratories, developing characterization tools based on new concepts, and increasing scientific collaboration. These recommendations from the Workshop were mirrored in the 2012 report Developing a Vision for the Infrastructure and Facility Needs of the Materials Community: NSF Materials 2022 from the NSF Mathematical and Physical Science Advisory Committee ${ }^{131}$

In recognition that some types of instrumentation are beyond the resources of individual institutions and have a wide user base, the NSF, the Department of Energy Basic Energy Sciences (DOE BES), and other federal agencies support national user facilities. These facilities are national assets, providing valuable access to state-of-the-art techniques; among these supported by the NSF are the Cornell High Energy Synchrotron Sources (CHESS) and the National High Field Magnetic Laboratory. An integrated network approach has also been adopted by the NSF in its support of the National Nanofabrication Infrastructure Network (NNIN) and the Center for High Resolution Neutron Science (CHRNS). The DOE supports more than 20 user facilities, including five light sources and three neutron sources; the National Institute of Standards and Technology (NIST) supports the NIST Center for Neutron Research user facility. These light and neutron sources are widely used by NSFsupported researchers, and where appropriate, joint funding among different federal agencies has supported the development of new instrumentation with broad applicability across federal R\&D agencies. All of these instruments open up new possibilities for characterization at the nanolength and microlength scales, especially for 3-D and 4-D measurements, and they provide answers to key MMN research questions that cannot be obtained in any other way. There is, however, a steep learning curve in performing research effectively at these facilities, from experiment planning and data analysis to its integration with modeling and computational materials science. To fill this gap, advanced graduate student and post-doc training for MMN research at light and neutron sources would complement existing DOE facility training and would be invaluable in increasing the impact of national facilities use. The emergence of multiple synchrotron facilities in the European Union, Australia, and Japan also highlights the trend toward centralization of instrumentation into national or regional user centers, requiring that investigators obtain critical resources for access, training, travel, and data analysis to take advantage of these opportunities. Given these trends, it is important that the NSF consider offering small-scale training and travel grants to graduate students, postdoctoral students, and junior faculty to use both national and 
international facilities. Not only would such grants prepare the future materials research workforce for increasingly complex MMN problems, but also they would also provide international research experiences and opportunities for collaboration to junior investigators. ${ }^{132}$

Connected to advanced materials instrumentation are the "big data" and instrument "forward modeling" challenges mentioned in the previous sections. As an example, 1 week of synchrotron experiments often produces so much data that as much as 6 months to 1 year is required for data processing and interpretation. The lack of an agreed-on materials taxonomy and metadata structure for a given instrument represents one barrier to efficient research progress. "In-line" data analysis and reconstruction with a well-defined "computational workflow" would permit more effective utilization of current instruments. ${ }^{132}$ In addition, there are still fundamental research issues that limit the merging and integration of data collected with different instruments on the same material. This is true even for data collected on a single sample when parallel "data channels" for different techniques on the same instrument sample different volumes. Solving such problems will substantially enhance the understanding of a wide spectrum of materials phenomena, as well as advance the goals of the MGI. As noted in the discussion of linking time and length scales in MMN research and the MGI, resolving these issues will require substantial multidisciplinary interactions, with strong participation by the NSF.

\section{EDUCATION AND WORKFORCE DEVELOPMENT}

Research in the area of MMN will continue to have a broad impact on a wide range of science and engineering challenges, but only if the future workforce is prepared to function in a rapidly changing environment. Foremost in the development of our future workforce is the training of students at the undergraduate and graduate levels. The Materials Science and Engineering curriculum has undergone a continuous and substantial evolution over the past four decades, evolving from early "materials specific" programs, such as metallurgical and ceramics engineering, to curricula that cover the full spectrum of materials. Undergraduate curricula now contain the hallmarks of materials science and engineering: structure, properties, processing, and performance. Required courses on the fundamentals in these areas tend to span the spectrum of materials classes without significant depth in specific systems, i.e., metals, ceramics, or soft materials.

At the workshop, there was widespread support for new, upper-level undergraduate courses that link length and time scales to processes and properties while integrating experiment, theory, and computation. Capstone design courses often focus on the engineering aspects of materials and emphasize the importance of design teams in that context. Similarly, interdisciplinary capstone teams could be developed to integrate experiment, theory, and computational materials science in a way that prepares undergraduates to work in MMN "research design" teams.

At the graduate level, incoming students often have undergraduate degrees in other fields, e.g., physics; chemistry; or electrical, mechanical, chemical, or biomedical engineering, and thus they have limited background in materials science and engineering. For this reason, core courses at the graduate level have become increasingly important for sustaining and defining the important role of materials science and engineering as a distinct discipline. Externally, the MGI and the need for integration of materials science with design of engineering systems motivate a stronger component of modeling and simulation at all length scales, as well as uncertainty quantification and informatics at both the graduate and undergraduate education level. Incorporating these topics into an already crowded and increasingly broad materials curriculum has proven to be challenging. ${ }^{133,134}$

Considerable time, effort, and creativity will be required to establish the computational expertise of MSE undergraduate and graduate students on equal footing with other engineering disciplines. Alternative curriculum development mechanisms are needed and the NSF can play a key role in this area via support for summer schools, workshops, and curriculum development activities. To make a lasting impact, a rigorous evaluation of new programs and approaches should be integral to these new activities. Professional societies can play an increasingly important role in convening and supporting such community-building activities for undergraduate and graduate students.

A strong future STEM workforce should naturally be diverse in gender, ethnicity, and geography. Historical trends in the diversity of materials science and engineering professionals from ASEE 2007-2011 data show essentially no change in the percentage of women and historically underrepresented groups in tenured and tenure-track faculty positions or receiving B.S. and Ph.D. degrees over that time period in the United States. ${ }^{135}$ Diversity in the student body will remain a challenge without role models; achieving diversity at the faculty level is critical for future progress but is difficult due to the long timeline of faculty careers. For this reason, mentoring of junior faculty is critical, and crossinstitutional mentoring could have major positive impacts. Because professional societies provide venues where such mentoring can naturally occur, they could play a key role in meeting this need.

The future health of the materials workforce will require professionals strongly educated in the fundamentals with additional skills for operating in 
interdisciplinary and international environments. In this context, research internships can substantially enhance the educational experience of both undergraduate and graduate materials students. Many large industrial organizations support U.S.based internship programs as a future investment in workforce. Unfortunately, these programs rarely offer similar opportunities internationally. In smaller industrial organizations, the cost of intern support often inhibits student hiring. Innovative programs that provide incentives to companies for hiring research interns could have substantial positive effects on students and their industry partners. At the graduate level, international research internships can provide access to unique instrumentation and foster other positive outcomes for graduate students, including long-term collaborations, patents, invitations for research lectures, and career opportunities, such as postdoctoral positions. Although the NSF has recognized the importance of international collaborations in programs such as the World Materials Network, the Partnership for International Research and Education, and the International Materials Institutes, continued strong support from all government agencies that support the materials mission will be needed to produce a long-term impact on the development of the materials science workforce.

\section{ACKNOWLEDGEMENTS}

The workshop was supported by the National Science Foundation Grant DMR-1240627. The authors acknowledge outstanding contributions in terms of oral presentations, discussion groups and selected excerpts within this document from workshop attendees, including: J. Allison, D. Bahr, S. Baker, E. Cerreta, N. Chawla, M. De Graef, D. Dimiduk, J. Erlebacher, J. Evans, K. Fahey, K. Findley, E. George, D. Gianola, P. Gumbsch, G.T. Gray, E. Holm, J.W. Jones, S. Kalidindi, S. Kang, D. Lados, R. LeSar, C. Levi, J. Lewandowski, D. Lewis, Q. Li, Z-Q. Liu, M. Manuel, E. Marquis, M. Mills, A. Mottura, B. Mueller, R. Odette, V. Ozolins, W. Reynolds, A. Rollett, M. Sangid, T. Schaedler, C. Schuh, D. Shih, A. Van der Ven, K. Vecchio, and P. Voorhees.

\section{REFERENCES}

1. I. Miller, D. Baker, D.C. Dunand, R. Dunin-Borkowski, B. Kabius, T. Kelly, S. Lozano-Perez, A. Misra, G.S. Rohrer, A.D. Rollett, M.L. Taheri, G.B. Thompson, M. Uchic, X.-L. Wang, and G. Was, J. Mater. Res. 26, 1341 (2011).

2. T.F. Kelly and M.K. Miller, Rev. Sci. Inst. 78, 031101 (2007).

3. P.A. Midley and M. Weyland, Ultramicroscropy 96, 413 (2003).

4. J.H. Sharp, J.S. Barnard, K. Kaneko, K. Higashida, and P.A. Midgley, J. Phys. Conf. Ser. 126, 012013 (2008).

5. P. Jornsanoh, G. Thollet, J. Ferreira, K. Masenelli-Varlot, C. Gauthier, and A. Bogner, Ultramicroscopy 111, 1247 (2011).

6. N.D. Browning, M.A. Bonds, G.H. Campbell, J.E. Evans, T. LaGrange, K.L. Jungjohann, D.J. Masiel, J. McKeown, S.
Mehraeen, B.W. Reed, and M. Santala, Curr. Opin. Solid State Sci. 16, 23 (2012).

7. H.F. Poulson, S.F. Nielsen, E.M. Lauridsen, S. Schmidt, R.M. Suter, U. Lienert, L. Margulies, T. Lorentzen, and D. Juul Jensen, J. Appl. Cryst. 34, 751 (2001).

8. W. Ludwig, A. King, P. Reischig, M. Herbig, E.M. Lauridsen, S. Schmidt, H. Proudhon, S. Forest, P. Cloetens, S. Rolland du Roscoat, J.Y. Buffière, T.J. Marrow, and H.F. Poulsen, Mater. Sci. Eng. A 524, 69 (2009).

9. J. Kaster, B. Harrer, G. Requena, and O. Brunke, $N D T \& E$ Int. 434, 599 (2010).

10. A.J. Kubis, G.J. Shiflet, D.N. Dunn, and R. Hull, Metall. Mater. Trans. A 35A, 1935 (2004).

11. M.D. Uchic, M.A. Groeber, D.M. Dimiduk, and J.P. Simmons, Scripta Mater. 55, 23 (2006).

12. G. Spanos, D.J. Rowenhorst, A.C. Lewis, and A.B. Geltmacher, MRS Bull. 33, 597 (2008).

13. M.D. Uchic, M. De Graef, R. Wheeler, and D.M. Dimiduk, Ultramicroscopy 109, 1229 (2009).

14. M.P. Echlin, A. Mottura, C.J. Torbet, and T.M. Pollock, Rev. Sci. Inst. 83, 023701 (2012).

15. D.J. Rowenhorst and P.W. Voorhees, Ann. Rev. Mater. Sci. 42, 105 (2012).

16. J.E. Spowart, H.M. Mullens, and B.T. Puchala, JOM 55, 35 (2003).

17. J.J. Williams, Z. Flom, A.A. Amell, N. Chawla, X. Xiao, and F. De Carlo, Acta Mater. 58, 6194 (2010).

18. E. Marquis, University of Michigan, private communication.

19. J. Bingert and G.T. Gray, private communication.

20. M. Mills, J. Kwon, and V. McCreary, unpublished research, Ohio State University (2012).

21. E.B. Gulsoy, J.P. Simmons, and M. De Graef, Scripta Mater. 60, 281 (2009).

22. G. Klimeck, M. McLennan, S. Brophy, G. Adams, and M. Lundstrom, IEEE Comput. Eng. Sci. (CISE) 10, 17 (2008).

23. L. Kaufman and H. Bernstein, Computer Calculation of Phase Diagrams with Special Reference to Refractory Metals (New York: Academic Press, 1970).

24. P.J. Spencer, CALPHAD 32, 1 (2008).

25. U.R. Kattner and C.E. Campbell, Mater. Sci. Tech. 25, 443 (2009).

26. J. Sato, T. Omori, K. Oikawa, I. Ohnuma, R. Kainuma, and K. Ishida, Science 312, 90 (2006).

27. T.M. Pollock, J. Dibbern, M. Tsunekane, J. Zhu, and A. Suzuki, JOM 62, 58 (2010).

28. T.M. Pollock, Science 238, 986 (2010).

29. A. Van der Ven, J.C. Thomas, Q. Xu, and J. Bhattacharya, Math. Comput. Simul. 80, 1393 (2010).

30. Z.-K. Liu, J. Phase Equilib. Diffus. 30, 517 (2009).

31. C. Wolverton, V. Ozolins, and M. Asta, Phys. Rev. B 69, 144109 (2004).

32. Q. Xu and A. Van der Ven, Acta Mater. 59, 1095 (2011).

33. A. Van der Ven, private communication.

34. P.Y. Chan, G. Tsekenis, J. Dantzig, K.A. Dahmen, and N. Goldenfeld, Phys. Rev. Lett. 105, 015502 (2010).

35. K.R. Elder, N. Provatas, J. Berry, P. Stefanovic, and M. Grant, Phys. Rev. B 75, 064107 (2007).

36. N. Goldernfeld, Athreya, and J.A. Dantzig, Phys. Rev. E 72, 020601 (2005).

37. J. Mellenthin, A. Karma, and M. Plapp, Phys. Rev. B 78, 184110 (2008).

38. M.E. McHenry, M.A. Willard, and D.E. Laughlin, Prog. Mater. Sci. 44, 291 (1999).

39. M. Willard, M. Daniil, and K.E. Knipling, Scripta Mater. 67,554 (2012).

40. T.M. Pollock, University of California Santa Barbara, unpublished figure.

41. S.R. Kalidindi, S.R. Niezgoda, and A.A. Salem, JOM 63, 34 (2011).

42. J. Miao, T.M. Pollock, and J.W. Jones, Acta Mater. 60, 2840 (2012).

43. L.J. Nelson, G.L.W. Hart, F. Zhou, and V. Ozolins, Phys. Rev. B 87, 035125 (2013). 
44. J.M. Rickman and R. LeSar, Scripta Mater. 54, 735 (2006).

45. N.M. Ghoniem, E.P. Busso, N. Kioussis, and H. Huang, Philos. Mag. A 83, 3475 (2003).

46. S. Papanikolaou, D.M. Dimiduk, W. Choi, J.P. Sethna, M.D. Uchic, C.F. Woodward, and S. Zapperi, Nature 490, 517 (2012).

47. C.Z. Zhou, S.B. Biner, and R. LeSar, Acta Mater. 58, 1565 (2010).

48. C.Z. Zhou, I.J. Beyerlein, and R. LeSar, Acta Mater. 59, 7673 (2011)

49. J.A. El-Awady, S.B. Biner, and N.M. Ghoniem, J. Mech. Phys. Sol. 56, 2019 (2008).

50. J.A. El-Awady, M. Wen, and N.M. Ghoniem, J. Mech. Phys. Solids 57, 32 (2009).

51. S.I. Rao, D.M. Dimiduk, T.A. Parthasarathy, M.D. Uchic, M. Tang, and C. Woodward, Acta Mater. 56, 3245 (2008).

52. J. Senger, D. Weygand, P. Gumbsch, and O. Kraft, Scripta Mater. 58, 587 (2008).

53. H. Tang, K.W. Schwarz, and H.D. Espinosa, Acta Mater. 55, 1607 (2007).

54. D. Weygand, M. Poignant, P. Gumbsch, and O. Kraft, Mater. Sci. Eng. A 483, 188 (2008).

55. H. Tang, K.W. Schwarz, and H.D. Espinosa, Phys. Rev. Lett. 100, 185503 (2008).

56. C. Motz, D. Weygand, J. Senger, and P. Gumbsch, Acta Mater. 57, 1744 (2009).

57. C.Z. Zhou and R. LeSar, Int. J. Plast. 30, 185 (2012).

58. P. Pant, K.W. Schwarz, and S.P. Baker, Acta Mater. 51, 3243 (2003)

59. B. von Blanckenhagen, P. Gumbsch, and E. Arzt, Mod. Simul. Mater. Sci. Eng. 9, 157 (2001).

60. B. von Blanckenhagen, P. Gumbsch, and E. Arzt, Philos. Mag. Lett. 83, 1 (2003).

61. B. von Blanckenhagen, E. Arzt, and P. Gumbsch, Acta Mater. 52, 773 (2004).

62. H.D. Espinosa, M. Panico, S. Berbenni, and K.W. Schwarz, Int. J. Plast. 22, 2091 (2006).

63. H.D. Espinosa, B.C. Prorok, and B. Peng, J. Mech. Phys. Sol. 52, 667 (2004).

64. R.S. Fertig III and S.P. Baker, Acta Mater. 58, 5206 (2010).

65. C.Z. Zhou and R. LeSar, Comput. Mater. Sci. 54, 350 (2012).

66. L.P. Kubin and G. Canova, Scripta Metall. Mater. 27, 957 (1992).

67. M. Verdier, M. Fivel, and I. Canova, Modell. Simul. Mater. Sci. Eng. 6, 755 (1998).

68. J. Bonneville and B. Escaig, Acta Metall. 27, 1477 (1979).

69. S. Rao, T.A. Parthasarathy, and C. Woodward, Philos. Mag. A 79, 1167 (1999).

70. S.I. Rao, D.M. Dimiduk, T.A. Parthasarathy, J. El-Awady, C. Woodward, and M.D. Uchic, Acta Mater. 59, 7135 (2011).

71. M.D. Sangid, T. Ezaz, H. Sehitoglu, and I. Robertson, Acta Mater. 59, 283 (2011).

72. M.D. Sangid, T. Ezaz, and H. Sehitoglu, Mater. Sci. Eng. A 542, 21 (2012).

73. S.R. Kalindindi, C.A. Bronkhorst, and L. Anand, J. Mech. Phys. Sol. 40, 537 (1992).

74. T.J. Turner, P.A. Shade, J.C. Schuren, and M.A. Groeber, Model. Simul. Mater. Sci. 21, 015002 (2013).

75. R. Pokharel, J. Lind, A.K. Kanjarala, R.A. Lebensohn, S.F. Li, P. Kenesei, R.M. Suter, and A.D. Rollett, Ann. Rev. Cond. Mat. Phys. 5, 317 (2014).

76. Integrated Computational Materials Engineering: A Transformational Discipline for Improved Competitiveness and National Security, Committee on Integrated Computational Materials Engineering and National Materials Advisory Board, Division of Engineering and Physical Sciences, National Research Council (Washington, DC: The National Academies Press, 2008).

77. J. Erlebacher, Johns Hopkins University, unpublished work (2012).

78. J. Erlebacher, Phys. Rev. Lett. 106, 225504 (2011).

79. I. McCue, J. Snyder, X. Li, Q. Chen, K. Sieradzki, and J. Erlebacher, Phys. Rev. Lett. 108, 225503 (2012).
80. T. Fujita, P. Guan, K. McKenna, X. Lang, A. Hirata, L. Zhang, T. Tokunaga, S. Arai, Y. Yamamoto, N. Tanaka, Y. Ishikawa, N. Asao, Y. Yamamoto, J. Erlebacher, and M.W. Chen, Nat. Mater. 11, 775 (2012).

81. M. Giesen, Prog. Surf. Sci. 68, 1 (2001).

82. Y. Ding, Y.-J. Kim, and J. Erlebacher, Adv. Mater. 16, 1897 (2004).

83. J. Rugolo, J. Erlebacher, and K. Sieradzki, Nat. Mater. 5, 946 (2006).

84. S.R. Brankovic, J.X. Wang, and R.R. Adzic, Surf. Sci. 474, L173 (2001).

85. Y.H. Liu, D. Gokcen, U. Bertocci, and T.P. Moffat, Science 338, 1327 (2012).

86. J.L. Stickney, Electroanal. Chem. 21, 75 (1999).

87. V.R. Stamenkovc, B. Fowler, B.S. Mun, G.F. Wang, P.N. Ross, C.A. Lucas, and N.M. Markovic, Science 315, 493 (2007).

88. M.J. Janik, C.D. Taylor, and M. Neurock, J. Electrochem. Soc. 156, B126 (2009).

89. X.H. Xia and Y.N. Xia, Nano Lett. 12, 6038 (2012).

90. H. Yu, M. Chen, P.M. Rice, S.X. Wang, R.L. White, and S.H. Sun, Nano Lett. 5, 379 (2005).

91. J.X. Wang, C. Ma, Y. Choi, D. Su, Y. Zhu, P. Liu, R. Si, M.B. Vukmirovic, Y. Zhang, and R.R. Adzic, J. Am. Chem. Soc. 133, 13551 (2011).

92. D. Margetis, Phys. Rev. B 76, 193403 (2007).

93. Y.C.K. Chen-Wiegart, S. Wang, Y.S. Chu, W.J. Liu, I. McNulty, P.W. Voorhees, and D.C. Dunand, Acta Mater. 60, 4972 (2012)

94. S.A. Policastro, J.C. Carnahan, G. Zangari, H. Bart-Smith, E. Seker, M.R. Begley, M.L. Reed, P.F. Reynolds, and R.G. Kelly, J. Electrochem. Soc. 157, C328 (2010).

95. P. Hirunsit and P.B. Balbuena, Surf. Sci. 603, 3239 (2009).

96. N. de Jonge and F.M. Ross, Nat. Nanotechnol. 6, 695 (2011).

97. G.R. Odette, M.J. Alinger, and B.D. Wirth, Ann. Rev. Mater. Sci. 38, 471 (2008)

98. A. Misra, M.J. Demkowicz, X. Zhang, and R.G. Hoagland, JOM 59 (9), 62 (2007).

99. K. Bourzac, MIT Tech. Rev. 20 (2011).

100. H.A. Murdoch and C.A. Schuh, Acta Mater. 61, 2121 (2013).

101. T. Chookajorn, H.A. Murdoch, and C.A. Schuh, Science 337, 951 (2012).

102. T. Frolov and Y. Mishin, Phys. Rev. Lett. 104, 055701 (2010).

103. T. Frolov, K.A. Darling, L.J. Kecskes, and Y. Mishin, Acta Mater. 60, 2158 (2012).

104. N.A. Mara, D. Bhattacharyya, P. Dickerson, R.G. Hoagland, and A. Misra, App. Phys. Lett. 92, 231901 (2008).

105. V. Radmilovic, C. Ophus, E.A. Marquis, M.D. Rossell, A. Tolley, A. Gautam, M. Asta, and U. Dahmen, Nat. Mater. 10, 710 (2011).

106. L. Popilevsky, V.M. Skripnyuk, Y. Estrin, A. Dahle, D. Mirabile Gattia, A. Montone, and E. Rabkin, Int. J. Hydrogen Energ. 1 (2013).

107. D.A. Molodov, T. Gorkaya, and G. Gottstein, J. Mater. Sci. 46, 4318 (2011).

108. M. Schamel, C. Schopf, D. Linsler, S.T. Haag, L. Hofacker, C. Kappel, H.P. Strunk, and G. Richter, Int. J. Mater. Res. 102, 828 (2011).

109. E. Shaffir, I. Reiss, and W.D. Kaplan, Acta Mater. 57, 248 (2009).

110. C.V. Thompson, Annu. Rev. Mater. Res. 42, 399 (2012).

111. J.P. Ye and C.V. Thompson, Acta Mater. 59, 582 (2011).

112. J.P. Ye and C.V. Thompson, Adv. Mater. 23, 1567 (2012).

113. D. Amram and E. Rabkin, Acta Mater. 61, 4113 (2013).

114. W.D. Kaplan, D. Chatain, P. Wynblatt, and W.C. Carter, J. Mater. Sci. 48, 5681 (2013).

115. E. Chason, Thin Solid Films 526, 1 (2012)

116. A.M. Vodnick and S.P. Baker, Acta Mater. 59, 5681 (2011).

117. Z.T. Trautt and Y. Mishin, Acta Mater. 60, 2407 (2012).

118. T. Frolov, W.J. Boettinger, and Y. Mishin, Acta Mater. 58, 5471 (2010). 
119. P. Sarobol, J.E. Blendell, and C.A. Handwerker, Acta Mater. 61, 1991 (2013).

120. F. Pei, N. Jadhav, and E. Chason, Appl. Phys. Lett. 100, 221902 (2012).

121. F. Pei, N. Jadhav, and E. Chason, JOM 64, 1176 (2012).

122. M.F. Ashby, J. Am. Ceram. Soc. 94, S3 (2011).

123. T.A. Schaedler, A.J. Jacobsen, A. Torrents, A.E. Sorensen, J. Lian, J.R. Greer, L. Valdevit, and W.B. Carter, Science 334, 962 (2011).

124. H.J. Rathbun, F.W. Zok, S.A. Waltner, C. Mercer, A.G. Evans, D.T. Queheillalt, and H.N.G. Wadley, Acta Mater. 54,5509 (2006).

125. D. Little, HRL Laboratories, LLC, private communication

126. G.L.W. Hart, S. Curtarolo, T.B. Massalski, and O. Levy, Phys. Rev. X 3, 041035 (2013).

127. M.D. Nielsen, V. Ozolins, and J.P. Heremans, Energy Environ. Sci. 6, 570 (2013).
128. L.Y. Chen, G. Richter, J.P. Sullivan, and D.S. Gianola, Phys. Rev. Lett. 109, 125503 (2012).

129. J.C. Zhao, X. Zheng, and D. Cahill, JOM 63, 40 (2011).

130. X. Zheng, D.G. Cahill, P. Krasnochtchekov, R.S. Averback, and J.-C. Zhao, Acta Mater. 55, 5177 (2007).

131. NSF Mathematical and Physical Science Advisory Committee, Developing a vision for the infrastructure and facility needs of the materials community: NSF Materials 2022, Subcommittee Report (Arlington, VA: NSF, 2012).

132. B. Fultz, J.J. Rehr, and S.J.L. Billinge, eds., Workflows for Computational Scattering Science 2013 (Arlington, VA: NSF, 2013).

133. K. Thornton, S. Nola, M. Asta, and G.B. Olson, JOM 61, 12 (2009).

134. G.B. Olson, Int. J. Eng. Ed. 17, 468 (2001).

135. L. Robinson, JOM 65, 7 (2013). 